\title{
Migration Velocity Analysis and Waveform Inversion
}

\author{
William W. Symes ${ }^{1}$ \\ 1 The Rice Inversion Project, Department of Computational and Applied Mathematics, \\ Rice University, Houston, TX 77005-1892 USA, email symes@caam.rice.edu
}

Accepted 200? ?? ??. Received 2007 Januari ??; in original form 2007 Januari ??

\begin{abstract}
SUMMARY
Least-squares inversion of seismic reflection waveform data can reconstruct remarkably detailed models of subsurface structure, and take into account essentially any physics of seismic wave propagation that can be modeled. However the waveform inversion objective has many spurious local minima, hence convergence of descent methods (mandatory because of problem size) to useful Earth models requires accurate initial estimates of long-scale velocity structure. Migration velocity analysis, on the other hand, is capable of correcting substantially erroneous initial estimates of velocity at long scales. Migration velocity analysis is based on prestack depth migration, which is in turn based on linearized acoustic modeling (Born or single-scattering approximation). Two major variants of prestack depth migration, using binning of surface data and Claerbout's survey-sinking concept respectively, are in widespread use. Each type of prestack migration produces an image volume depending on redundant parameters, and supplies a condition on the image volume which expresses consistency between data and velocity model, hence a basis for velocity analysis. The survey-sinking (depth-oriented) approach to prestack migration is less subject to kinematic artifacts than is the binning-based (surface-oriented) approach. Because kinematic artifacts strongly violate the consistency or semblance conditions, this observation suggests that velocity analysis based on depth-oriented prestack migration may be more appropriate in kinematically complex areas. Appropriate choice of objective (differential semblance) turns either form of migration velocity analysis into an optimization problem, for which Newton-like methods exhibit little tendency to stagnate at nonglobal minima. The extended modeling concept links migration velocity analysis to the apparently unrelated waveform inversion approach to estimation of Earth structure: from this point of view, migration velocity analysis is a solution method for the linearized waveform inversion problem. Extended modeling also provides a basis for a nonlinear generalization of migration velocity analysis. Preliminary numerical evidence suggests a new approach to nonlinear waveform inversion which may combine the global convergence of velocity analysis with the physical fidelity of model-based data fitting.
\end{abstract}

Key words: Velocity analysis, waveform inversion, least squares

\section{INTRODUCTION}

Seismic waveform inversion is a synonym for model-based fitting of waveform (sampled hydrophone or geophone) data. It is often formulated as the least squares problem of reducing the energy in the difference between predicted and observed data to a minimum, by varying the model on which the prediction is based. This output least squares formulation will be taken as a synonym for waveform inversion throughout the following discussion.

The model-based data-fitting approach to inference of Earth structure from geophysical data is conceptually attractive, and has a long and productive history in the Earth sciences. Its influence on reflection seismology has nonethe- less been limited by two major impediments. The first is the computational intensity of reflection seismogram modeling, especially in 3D. Output least squares inversion requires full wavefield modeling and various associated computational tasks, and until recently these computations were beyond reach at industrially or scientifically relevant scales. This computational obstacle is gradually fading, due to continued steady advances in computer hardware performance and algorithmic improvements in modeling.

The second impediment is more fundamental. Leastsquares estimates, or rather their prestack depth migration approximations, have become the preeminent tool for estimating short, or wavelength, scale Earth structure given a long scale model. However the least squares principle has 
proven poorly suited to inference of long scale structure (often called the macromodel), as we shall explain below. Since accurate knowledge of the long scale structure is prerequisite to successful estimation of short scale structure, this circumstance has strictly limited the straightforward application of waveform inversion. Instead, industrial seismology has developed a suite of migration velocity analysis techniques, apparently completely unrelated to waveform inversion, for the estimation of Earth macromodels.

The central thesis of this paper is that migration velocity analysis is in fact an approach to solving a partially linearized variant of the waveform inversion problem (linear in short scales, nonlinear in long scales). The conceptual link between migration velocity analysis and waveform inversion is the notion of extended model, in which the modeling of wavefields is extended to nonphysical models depending on redundant parameters. I will show that both major variants of migration velocity analysis, based on either data binning (surface oriented or "Kirchhoff") and survey-sinking (depthoriented or "wave equation") variants of prestack depth migration, amount to the solution of waveform inversion problems for appropriate extended models. These models use linearized or Born modeling at short scales, but include the full nonlinear (kinematic) effect of the macromodel - hence "partially linearized". The kinematic properties of surfaceoriented and depth-oriented prestack migrations and their associated extended models may be quite different, and these kinematic disparities may have some consequences for migration velocity analysis, as I shall explain.

These observations provides a conceptual framework for recent attempts to quantify and automate migration velocity analysis. Moreover, the viewpoint developed here also suggests that the extended modeling principle might be applied directly to nonlinear waveform inversion, and that this extended formulation might overcome the fundamental obstacle to effective waveform inversion in reflection seismology. I will present some preliminary evidence concerning the behaviour of nonlinear extended inversion, suggesting that a nonlinear analogue of the semblance conditions of migration velocity analysis may distinguish data-consistent from data-inconsistent models. I will also discuss the remaining challenges, both conceptual and computational, to implementation of this approach to nonlinear inversion.

Except for a few allusions, this paper does not include any discussion of traveltime tomography. In fact, reflection traveltime tomography is widely used as a velocity analysis method. Common reflection tomography techniques exploit many of the concepts discussed in this paper, and are highly automated in current industry practice. The aim of this paper, however, is to describe methods which estimate Earth models directly from waveform data, without the data reduction that is the necessary first stage for any version of traveltime tomography.

Much of this paper concerns mathematical formulations of various versions of waveform inversion. I shall minimize mathematical formalism in this discussion, subject to introducing sufficient concepts and notation to support the main ideas. Extensive references provide the reader with access to many mathematical details not developed with full precision in the following pages. The citations cannot be complete, as the literature on these topics is extensive. I have tried to cite a representative sample, from which the interested reader can follow further leads.

The next two sections present an overview of output least squares inversion and migration velocity analysis. The fourth section describes the extended modeling concept and its partially linearized variant, and establishes the link between migration velocity analysis and output least squares inversion. The fifth section describes the extended model approach to fully nonlinear output least squares inversion and describes some initial numerical experiments which illustrate some of its properties. The paper ends with a discussion of prospects for further development of this nonlinear migration velocity analysis - waveform inversion hybrid.

\section{WAVEFORM INVERSION}

An abstract setting for geophysical inversion relates the model space $M$ - a set of possible models of Earth structure - to the data space $D$ through the forward map or prediction operator $F: M \rightarrow D$. The simplest version of data fitting inversion asks that $m \in M$ be chosen to minimize the mean square data misfit between the forward map output $F[\mathrm{~m}]$ and an observed datum $d \in D$ : that is,

$$
\min _{m} J_{\mathrm{OLS}}[m, d] \equiv \frac{1}{2}\|F[m]-d\|^{2}
$$

in which the symbol $\|\cdot\|$ stands for a (Hilbert) norm in the data space $D$. Generally $M$ is actually an admissible subset of a vector space of functions, incorporating bounds on values (velocity is positive, etc. etc.) and perhaps other physically or mathematically motivated constraints. I will use the notation $M$ to denote both this set and its ambient vector space, relying on context to distinguish the two, and refer to both as "model space".

In practice, geophysical inverse problems tend to be both overdetermined and underdetermined: that is, the minimum value of $J_{\text {OLS }}$ is unlikely to be zero (data is inconsistent), and many different models $m$ may come within a reasonable tolerance of the best fit level achievable (data is inadequate).

Data fitting inversion has a long and productive history, and geoscientists have developed considerable sophistication in understanding the implications of data incompleteness and inconsistency. The excellent papers of Backus \& Gilbert (1968, 1970), Jackson (1972, 1976, 1979) and Robinson \& Treitel (1980) continue to reward the reader. Parker (1977), Tarantola (1987), and Lines \& Treitel (1984) provide excellent overviews of theory and application.

The application of data fitting inversion to reflection seismology dates at least to the pioneering work of Bamberger, Chavent, and Lailly in the late 70's (Bamberger et al. 1977, 1979) on the one-dimensional model problem. In contrast to much subsequent work, this study illustrated the physical and mathematical consequences of model space metric definition.

The simplest model which exhibits the basic kinematic and dynamic complexity of field reflection seismograms is constant-density acoustics, based on the causal initial value problem for the wave equation

$$
\left(\frac{1}{v^{2}(\mathbf{x})} \frac{\partial^{2}}{\partial t^{2}}-\nabla^{2}\right) u\left(t, \mathbf{x} ; \mathbf{x}_{s}\right)=f\left(t, \mathbf{x} ; \mathbf{x}_{s}\right)
$$




$$
u(t, \cdot ; \cdot) \equiv 0, t<<0,
$$

in which $\mathbf{x}$ denotes position within a model of the Earth, $v(\mathbf{x})$ is the acoustic velocity field, $u\left(t, \mathbf{x} ; \mathbf{x}_{s}\right)$ is the acoustic potential, and $f\left(t, \mathbf{x} ; \mathbf{x}_{s}\right)$ is a body force representation of the energy source, indexed by source location $\mathbf{x}_{s}$.

The model space $M$ is a set of possible velocity fields $v$, i.e. $m=\{v(\mathbf{x})\}$. The data space $D$ consists of samples of pressure at a collection of receiver locations $\mathbf{x}_{r}$ possibly depending on the source location $\mathbf{x}_{s}$, over a time interval $t_{\text {init }} \leq t \leq t_{\text {final }}$. The energy source (right-hand side in the wave equation (2) may be regarded as known, or estimated along with the velocity. $D$ is regarded as a Hilbert space, equipped with some version of the $L^{2}$ norm. Thus the forward map $F: M \rightarrow D$ is defined by

$$
F[v]\left(t, \mathbf{x}_{r} ; \mathbf{x}_{s}\right)=\frac{\partial u}{\partial t}\left(t, \mathbf{x}_{r} ; \mathbf{x}_{s}\right)
$$

Throughout this paper, I shall alternate between notation appropriate to the acoustic model $(v \in M, F[v], \ldots)$, when discussing concrete features of acoustic scattering and data processing concepts based on it, and the general inverse theory framework introduced in this section $(m \in$ $M, F[m], \ldots)$, when more generality is appropriate.

In order to resolve features of geologic interest, the dimension of the model space $M$ must be rather large, on the order of $10^{4}-10^{6}$ in $2 \mathrm{D}$, one or more orders of magnitude greater for 3D. Accordingly only iterative optimization methods with convergence rates more or less independent of model space dimension are feasible for these problems. In practice, this means Newton's method and various relatives. Chavent had already shown in the 1970's how to compute the gradient of functionals of solutions of time dependent problems, using the so-called adjoint state method borrowed from control theory (Chavent \& Lemmonier 1974). Applied to the wave equation (2), this principle becomes a version of prestack reverse time migration (Lailly 1983, 1984; Tarantola 1984).

Application of Newton-like optimization to this acoustic inverse problem, and to more complex problems of which it is a special case, has revealed a surprising obstacle: under prototypical conditions of acquisition geometry and bandwidth, the output least squares objective (1) appears to possess many stationary points ("local minima"), most of them quite far from the global minimum even for noise-free data (in which case the global minimum occurs at the model generating the data). Tarantola and coworkers provided an early example of this pathology (Gauthier et al. 1986), and many others have since been constructed (Kolb et al. 1986; Santosa \& Symes 1989; Shin \& Min 2006). While many attempts have been made to work around this obstacle (Tarantola et al. 1990; Mosegard \& Tarantola 1991; Sen \& Stoffa 1991a,b; Clément \& Chavent 1993; Jin \& Madariga 1994; Bunks et al. 1995; Plessix et al. 1995, 1999; Shin \& Min 2006), all results to date are consistent with the conclusion that

Under prototypical conditions of acquisition geometry and bandwidth, the output least squares objective function is strongly multimodal, and unsuitable for global minimization via Newton-like optimization methods.

The factors which appear to drive the pathological behaviour of output least squares for the acoustic inverse prob- lem are (1) reflection geometry, i.e. sources and receivers separated from the scattering region by a hyperplane, and (2) bandlimitation of the recorded signals, in particular the absence of very low frequency energy. Transmitted wave data tends to reduce somewhat the proclivity of local minima to appear, as Tarantola et al. already observed (Gauthier et al. 1986). Mora (1988) gave a partial explanation of this tendency, and Pratt and coworkers (Pratt 1999; Pratt \& Shipp 1999; Sirgue \& Pratt 2004; Brenders \& Pratt 2006a,b) have exploited this observation to devise functional output least squares inversion for diving wave and crosshole geometries.

The oscillatory nature of field data, i.e. its lack of low frequencies, appears to pose a very serious obstacle to successful output least squares inversion in the reflection configuration. It has been known for decades that the impedance as a function of vertical traveltime in a layered acoustic medium could be reconstructed from the impulsive normal incidence plane wave trace. That is, for these essentially 1D problems, inversiion for impedance is well-posed provided that all frequency components down to $0 \mathrm{~Hz}$ are available in the data, (Goupillaud 1961; Symes 1980; Santosa \& Schwetlick 1982; Bube \& Burridge 1983; Symes 1983, 1986a; Sacks \& Santosa 1987; Carazzone \& Srnka 1989). Low-cut filtering renders the reconstruction ambiguous (Pao et al. 1984; Gray \& Symes 1985; Santosa \& Symes 1989). For several dimensional problems, numerical explorations indicate that impulse responses (i.e. sampling of Green's functions) appear to uniquely determine constant-density acoustic models (velocity fields) via output least squares inversion (Bunks et al. 1995; Shin \& Min 2006), although no rigorous mathematical argument to that effect has yet been put forth, under the natural conditions of seismic modeling.

Numerical evidence of spurious stationary points in the absence of low frequency energy $(<3-5 \mathrm{~Hz}$, other scales being typical of exploration seismology) is quite strong, both direct (plots of objective vs. velocity, eg. Symes \& Carazzone (1992)) and indirect (failure of Newton-like optimization algorithms to achieve convergence to global minimum (Gauthier et al. 1986; Tarantola et al. 1990; Shin \& Min 2006)). While no proof exists, in the mathematical sense, of the existence of spurious minima, the $\mathrm{PhD}$ thesis of Chauris (2000) suggests a plausible explanation. In the context of migration velocity analysis (discussed later in this paper), Chauris observes that unrelated events in prestack image volumes for correct and incorrect velocities may be tangent, leading to constructive interference in the stack and anomalously high stack power at kinematically incorrect velocities. Chauris illustrates the resulting appearance of spurious local maxima of stack power. In the context of data fitting, it seems reasonable to expect that similar tangencies between predicted and observed data events may occur at incorrect velocities, leading to anomalously good local data fit and thus spurious local minima of the mean-square error.

Spurious stationary points do not appear to exist at models whose kinematics are sufficiently close to those of the data. Thus a sufficiently good initial guess, providing the predicted data with events whose geometry closely matches that of the observed data, should permit convergence of Newton-like iteration to a satisfactory global minimum. Such convergence has been observed many times (Cao et al. 1990; Crase et al. 1990; Bunks et al. 1995; Plessix et al. 1999; Shin \& Min 2006; Brenders \& Pratt 2006a). In 
some cases the initial estimate has been provided by traveltime tomography, in others by migration velocity analysis (described in the next section). The multiscale inversion of Bunks et al. (1995) can even be viewed as providing a reasonable initial guess for seismic band inversion by first inverting very low frequency data (significant energy as low as $0.25 \mathrm{~Hz}$, in the example of Bunks et al. (1995)). Unfortunately no a priori assessment of initial estimate adequacy seems possible: it is quite common to fail to converge to a useful final estimate from an apparently reasonable initial estimate.

Granted that output least squares inversion by Newtonlike optimization converges to a best-fitting model, provided that a sufficiently good initial guess may be procured, it is natural to ask whether in fact (field) seismic data can be fit by such a model-driven process. Few studies of waveform inversion have modeled enough of the important physics of seismic wave generation and propagation to answer this question. Minkoff's 1995 thesis (Minkoff \& Symes 1997) is one exception to this pattern. Minkoff used a migration velocity analysis derived initial model estimate together with model-based attenuation parameter estimate and linearized viscoelastic modeling to estimate directionally dependent source wavelet and perturbational elastic parameters by output least squares inversion. By taking all apparently relevant physics into account, she was able to fit $90 \%$ of the energy in the small plane wave data set used in the study. Furthermore, only when all relevant physics was incorporated in the model did the inversion yield lithologically correct predictions, validated by logging (Minkoff \& Symes 1997).

While more studies are needed incorporating this degree of physical realism and falsification (validation by independent criteria) design, Minkoff's thesis results give some limited confidence that waveform data fitting can indeed yield valid and highly detailed inference about the Earth's interior. The principal obstacle to use of this technique is provision of a reliable starting model, in the reflection configuration and with typical seismic bandwidth. Since the long scale components in the compressional velocity are critically important in this starting model, it is possible to study the issue to some extent within the context of constant density acoustics. One of the industry techniques for constructing seismic propagation models, migration velocity analysis, appears to be able to produce large velocity updates directly from data with prototypical time, length, and frequency scales.

\section{LINEARIZATION}

The industry standard approach to determination of Earth structure from seismic reflection data relies upon the linearized model of acoustic scattering (single scattering, Born approximation), which results from applying first order perturbation theory to equation (2):

$$
\begin{gathered}
\left(\frac{1}{v^{2}(\mathbf{x})} \frac{\partial^{2}}{\partial t^{2}}-\nabla^{2}\right) \delta u\left(t, \mathbf{x} ; \mathbf{x}_{s}\right)=\frac{2 \delta v(\mathbf{x})}{v^{3}(\mathbf{x})} \frac{\partial^{2} u}{\partial t^{2}}\left(t, \mathbf{x} ; \mathbf{x}_{s}\right) \\
\delta u(t, \cdot ; \cdot) \equiv 0, t<<0
\end{gathered}
$$

The linearized forward map or Born scattering operator $D F$ maps the tangent space $M \times M$ of (reference model, model perturbation) pairs to the data space $D$. For acoustic modeling, $D F$ is defined by

$$
(D F[v] \delta v)\left(t, \mathbf{x}_{r} ; \mathbf{x}_{s}\right)=\frac{\partial \delta u}{\partial t}\left(t, \mathbf{x}_{r} ; \mathbf{x}_{s}\right) .
$$

The reference velocity model $v$ is supposed to account for the kinematics of data, hence the large scale stucture of the Earth. Thus it is appropriate to assume $v$ to be smooth or slowly varying, or at least piecewise smooth, on the scale of a wavelength. The perturbation $\delta v$ is presumed to carry the wavelength scale features of Earth structure, and so is oscillatory. The notation is chosen to remind the reader that $D F$ depends nonlinearly on the reference model $(v$ in this case) and linearly on the model perturbation $(\delta v)$.

Many of the statements to be made in the sequel about the behavior of the linearized operator $D F[v]$ rely on additional constraints being imposed on the velocity model $v$, such as explicit upper and lower bounds and bounds on spatial gradients. These additional requirements define appropriate admissible sets of velocity models. I will not specify admissible sets explicitly in this paper, but will tacitly assume that restriction of $D F$ to an admissible set of velocities is in force.

Existence of the derivative $D F$ under very general circumstances, as a bounded operator on suitable Hilbert spaces, is established by Stolk (2000a), see also FernandezBerdaguer et al. (1996). Apparently the first order Taylor polynomial remainder $F[v+\delta v]-F[v]-D F[v] \delta v$ (otherwise put, the error in the Born approximation) is anomalously small when $v$ is sufficiently smooth (bounds on derivatives) and $\delta v$ oscillatory (sufficiently small low-order moments). On the other hand, this linearization error is large when the background contains rapid variations (is not smooth on wavelength scale) and the perturbation has low frequency components. That is, the Born approximation is considerably more accurate when reference and perturbation velocities are separated in scale (long for reference, short for perturbation), considerably less accurate otherwise. Since the linearization error is related to the degree of nonlinearity of the least-squares misfit function, the violation of this scale dichotomy in velocity updates spanning all scales is a partial explanation for the failure of gradient methods in waveform inversion. Considerable numerical evidence supports this point of view (Symes 1995), but theoretical justification exists only for the special case of 1D modeling (Lewis \& Symes 1991; Symes 1991a).

The linearized or Born scattering operator $D F[v]$, for smooth background velocity $v$, has been studied extensively over the last twenty years, beginning with the pioneering work of Beylkin (1985), Bleistein (1987), and Rakesh (1988). As the wave operators appearing in (2) and (4) have smooth coefficients, oscillatory solutions are well approximated by geometric optics, and $D F[v]$ by an oscillatory integral operator (generalized Radon transform, (Beylkin 1985)). These approximations underly an analysis of the linearized output least squares problem

$$
\operatorname{minimize} \frac{1}{2}\|D F[v] \delta v-(d-F[v])\|^{2} \text { over } \delta v \in M .
$$

A minimizer of the function (6) of minimal length is a socalled pseudoinverse solution, written $\delta v=D F[v]^{\dagger}(d-F[v])$ and well-approximated by the regularized least-squares so- 
lution

$$
\delta v \simeq\left(D F[v]^{*} D F[v]+\lambda I\right)^{-1} D F[v]^{*}(d-F[v])
$$

for small values of $\lambda$.

The essential properties of $D F[v]$ and its least squares problem follow from the modern theory of oscillatory integrals, and are easiest to state when it is assumed acquisition geometry is complete, i.e. that essentially all source-receiver azimuths are available, that sampling is dense enough to be neglected, and that the source is impulsive: $f\left(\mathbf{x}, t ; \mathbf{x}_{s}\right)=$ $\delta(t) \delta\left(\mathbf{x}-\mathbf{x}_{s}\right)$. Then

$D F[v]$ is "almost unitary", in the sense that its adjoint or transpose $D F[v]^{*}$ differs from its (pseudo)inverse by dip-dependent scaling and filtering;

If the data $d$ is nearly consistent with the Born scattering model, $d \simeq F[v]+D F[v] \delta v$ for smooth $v$ and oscillatory $\delta v$, then the least squares gradient $D F[v]^{*}(d-F[v])$ is an image of $\delta v$, in the sense of having the same oscillatory components or locations of rapid change ("reflectors"), except for dip-dependent scaling and filtering.

The almost-unitary property of $D F[v]$ on oscillatory data was established by Beykin and Rakesh in the cited references, under the assumption that all sources and receivers are connected to all possible scattering points (support of $\delta v$ ) by unique rays of geometric optics. This latter assumption was gradually relaxed through the work of Ten Kroode et al. (1998), Nolan \& Symes (1997), and Stolk (2000a,b). In particular, Stolk shows that the almost-unitary property of $D F[v]$ is a generic: if $v$ doesn't have it, then arbitrarily small perturbations of $v$ do (for $2 \mathrm{D}$ acoustics - the analogous property is conjectured to hold in $3 \mathrm{D}$, but has not yet been proven). The normal operator $D F[v]^{*} D F[v]$ acts by dip-dependent scaling and filtering: it is a so-called pseudodifferential operator, for generic smooth background velocities $v$. In particular, application of the normal operator does not affect the location or orientation of highly oscillatory or short-scale features of its argument. This fact is responsible for the almost-unitary nature of the linearized map, and implies that its adjoint $D F[v]^{*}$ is a structural imaging operator, in fact a version of prestack depth migration. This relation of linearized inversion to prestack depth migration was noted by Lailly (1983, 1984) and Tarantola (1984). A good reference for the definition and properties of pseudodifferential operators is Taylor (1981).

Nolan (Nolan \& Symes 1997) also shows how the above statements must be modified when the acquisition geometry is incomplete, i.e. idealized narrow azimuth surveys. A number of researchers have generalized these results to various elastic settings of the seismic inverse problem (Beylkin \& Burridge 1990; de Hoop \& Bleistein 1997; Burridge et al. 1998; de Hoop \& Stolk 2002). The details are naturally more complex, but the gist is as called out above: the linearized scattering operators are almost unitary in general, and their normal operators act by dip-dependent scaling and filtering, provided full bandwidth and spatially complete data is available. approximate solutions of linearized output least squares problem can be written as oscillatory integrals, convenient for computation.

\section{MIGRATION VELOCITY ANALYSIS}

Solution of the linearized inverse problem, or approximate solution (prestack depth migration), requires a velocity (reference) model $v$, which must also be estimated somehow from the data. The geophysical prospecting industry has developed a set of techniques for velocity estimation, called migration velocity analysis (Yilmaz 2001). In contrast to prestack depth migration itself, migration velocity analysis appears at first glance to have little to do with output least squares inversion.

migration velocity analysis consists of two steps: given a velocity model,

- produce an image volume, containing the image (approximate linearized inverse) but also depending on one or more redundant parameters;

- apply an imaging condition to produce an image and, as a by-product, an updated velocity.

In principle, these steps may be carried out a number of times.

\section{Image Volume Construction}

Two distinct definitions of image volume are in common use. Both are most easily formulated with the aid of the solution operator of the linearized acoustic problem (4). Using the Green's function $G$ of the reference medium, i.e. the causal solution of equation (2) with $f\left(t, \mathbf{x} ; \mathbf{x}_{s}\right)=\delta\left(\mathbf{x}-\mathbf{x}_{s}\right) \delta(t)$, one can write an integral representation for the solution of (4), evaluated at a receiver position $\mathbf{x}_{r}$ :

$$
\begin{gathered}
\delta u\left(t, \mathbf{x}_{r} ; \mathbf{x}_{s}\right)= \\
\frac{\partial^{2}}{\partial t^{2}} \int d \mathbf{x} \int d \tau G\left(t-\tau, \mathbf{x} ; \mathbf{x}_{r}\right) \frac{2 \delta v(\mathbf{x})}{v^{3}(\mathbf{x})} G\left(\tau, \mathbf{x} ; \mathbf{x}_{s}\right)
\end{gathered}
$$

The physical meaning of equation (7) may be read from the integrand, from right to left. The first Green's function propagates a wave from the source at $\mathbf{x}_{s}$ to the scattering point $\mathbf{x}$. There, the wave interacts with the material heterogeneity represented by $\delta v$. This interaction is scaled in the space domain by $v^{-3}$ and in the frequency domain by $\partial^{2} / \partial t^{2}$. This scaling derives from the structure of the virtual Born source (right-hand-side of equation (4)), and correctly dimensions the integrand for integration over a volume. Finally, the second Green's function propagates the signal generated by the wave-heterogeneity interaction from the scattering point $\mathbf{x}$ to the receiver $\mathbf{x}_{r}$.

In view of the definition (5), the integral representation (7) shows $D F[v]$ to be an integral operator with kernel

$$
\frac{\partial^{3}}{\partial t^{3}} \int d \tau G\left(t-\tau, \mathbf{x} ; \mathbf{x}_{r}\right) G\left(\tau, \mathbf{x} ; \mathbf{x}_{s}\right) \frac{2}{v^{3}(\mathbf{x})}
$$

That is, one can view this expression as the "matrix" of $D F[v]$, with $\left(d t, \mathbf{x}_{r}, \mathbf{x}_{s}\right)$ and $\mathbf{x}$ playing the roles of row and column indices respectively. Thus the adjoint or transpose linearized operator has the integral representation

$$
\begin{gathered}
D F[v]^{*}(d-F[v])(\mathbf{x})=-\frac{2}{v^{3}(\mathbf{x})} \int d \mathbf{x}_{s} \int d \mathbf{x}_{r} \int d t \int d \tau \times \\
G\left(t-\tau, \mathbf{x} ; \mathbf{x}_{r}\right) G\left(\tau, \mathbf{x} ; \mathbf{x}_{s}\right) \frac{\partial^{3} d}{\partial t^{3}}\left(t, \mathbf{x}_{r} ; \mathbf{x}_{s}\right)
\end{gathered}
$$


As noted above the output of $D F[v]^{*}$ is an image of the subsurface, having approximately correct short-scale features as $\delta v$ when $d \simeq D F[v] \delta v$. I will denote the output by $I(\mathbf{x}) \equiv D F[v]^{*}(d-F[v])$.

The surface oriented image volume definition introduces a surface acquisition parameter as the redundant degree of freedom, and limits the integration in (8) to common value (level) sets of this parameter (i.e. to gathers). For example, the common offset image volume $I_{\mathrm{CO}}$ is constructed by restricting (8) to common offset gathers: for (vector) half-offset h, define

$$
\begin{gathered}
I_{\mathrm{CO}}(\mathbf{x}, \mathbf{h})=-\frac{2}{v^{3}(\mathbf{x})} \int d \mathbf{x}_{s} \int d t \int d \tau \times \\
G\left(t-\tau, \mathbf{x} ; \mathbf{x}_{s}+2 \mathbf{h}\right) G\left(\tau, \mathbf{x} ; \mathbf{x}_{s}\right) \frac{\partial^{3} d}{\partial t^{3}}\left(t, \mathbf{x}_{s}+2 \mathbf{h} ; \mathbf{x}_{s}\right) .
\end{gathered}
$$

In practice, this integral is further averaged over bins in $\mathbf{h}$, or over an annulus in $\mathbf{h}$ to create a function of (scalar) offset $h=|\mathbf{h}|$.

Synthesis of plane wave response leads to a similar definition of plane (or conical) wave image volume (Treitel et al. 1982; Duquet \& Lailly 2006). An even less straightforward variant of this concept is the common scattering angle volume (Xu et al. 2001; Stolk 2000a,b; Brandsberg-Dahl et al. 2003), in which the integral (8) is recast as an integral in phase space and subintegrals formed over data subsets (in phase space) characterized by common scattering angle.

All of the surface oriented image volume constructions share a common defect, in that kinematic artifacts may form when the velocity structure is sufficiently complex that significant energy can propagate along multiple paths connecting source, receiver, and scattering points (Nolan \& Symes 1997; Brandsberg-Dahl et al. 2003; Stolk \& Symes 2004). These artifacts arise because the restricted integrals in volume formation rules such as (9) do not restrict the slowness component of rays contributing to the image output, corresponding to the gather direction. For example, integration over a common offset gather implicitly combines image contributions propagating along all rays sharing a common midpoint ray parameter, but with possibly different offset ray parameters (difference of source, receiver horizontal ray parameters). Therefore energy arriving in the data along one pair of rays can migrate to a different point in the image volume along a different pair of rays, with different offset ray parameter. Similar image ambiguity may arise in any of the surface-oriented image volumes defined above, including common scattering angle volumes. The artifact events produced in this way can easily be as energetic as the events corresponding to actual reflectors. Their presence has serious consequences for the use of this type of image volume in velocity analysis, as explained below.

The second type of image volume definition introduces additional degrees of freedom by means of spatial and/or temporal shifts applied to the integrands in (8). The version of this depth oriented image volume appropriate to $s u b$ surface vector half-offset $\mathbf{h}$ (note: not the same as sourcereceiver offset vector used in the definition of $I_{\mathrm{CO}}$ !) is

$$
I_{\mathrm{SS}}(\mathbf{x}, \mathbf{h})=\frac{-2}{v(\mathbf{x}+\mathbf{h}) v^{2}(\mathbf{x}-\mathbf{h})} \int d \mathbf{x}_{s} \int d \mathbf{x}_{r} \int d t \int d \tau \times
$$

$$
G\left(t-\tau, \mathbf{x}+\mathbf{h} ; \mathbf{x}_{r}\right) G\left(\tau, \mathbf{x}-\mathbf{h} ; \mathbf{x}_{s}\right) \frac{\partial^{3} d}{\partial t^{3}}\left(t, \mathbf{x}_{r} ; \mathbf{x}_{s}\right) .
$$

Claerbout (Claerbout 1971; Claerbout \& Doherty 1972) introduced essentially this definition, with the exceptions that (i) the factors of $v$ and the $t$ derivatives were missing - the reasons for their presence in our formulation will become apparent below; (ii) $\mathbf{h}$ is constrained to be horizontal, and that remains the most common variant. Indeed, Claerbout proposed viewing this image volume as the result of sinking the survey, with "sunken" sources and receivers $(\mathbf{x}-\mathbf{h}$ and $\mathbf{x}+\mathbf{h}$ respectively) on planes of increasing depth (Claerbout 1985). Claerbout also showed how to build this image volume by means of one-way wavefield extrapolation from surface data (as opposed to evaluation of an integral like the above). It is also possible to use two-way wavefield extrapolation, i.e. solution of the acoustic wave equation (2) and related computations, to accomplish this task, via a variant of reverse time migration (Biondi \& Shan 2002; Symes 2002). Nonhorizontal subsurface offset may find constructive uses (Biondi \& Symes 2004; Biondi \& Shan 2002; Symes 2002), as can time (as opposed to spatial offset) shifts (Sava \& Fomel 2005). Scattering angle can also be used as the redundant parameter (Prucha et al. 1999; Rickett \& Sava 2002; Sava \& Fomel 2003).

The computational cost of depth oriented image volume formation can be considerable. The integral on the righthand side of equation (10) can be interpreted as the crosscorrelation at a range of spatial shifts of the field produce by the source $\left(G\left(\tau, \mathbf{x}-\mathbf{h} ; \mathbf{x}_{s}\right)\right.$, under the integral sign $)$, and the field produced by propagating the recorded data $d$ backwards in time (integral over $\mathbf{x}_{r}, t$ of the remaining factors), followed by a final summation over sources. This shot-profile profile organization of the computation is the only one available for the reverse time approach, and is one of the two common techniques employing one-way propagators (Biondi \& Palacharla 1996; Biondi 2003). The additional computational load due to the cross-correlations can easily dwarf that due to propagation. A commonly used workaround is to avoid computing the full image volume. For velocity analysis, as reviewed below, a relatively small fraction of the image volume suffices to constrain the velocity estimate. Thus much of the crosscorrelation load can be avoided, and the cost of the partial image volume formation approaches that of image formation alone.

Surface oriented image volume formation is often termed "Kirchhoff prestack depth migration". It is usually carried out via the formula (9) with the Green's function $G$ replaced by its asymptotic (ray-theoretic) approximation. The differential equation approach introduced by Claerbout (1971) is the predominant method for depth oriented image volume production, hence the common usage "prestack wave equation migration" for this technique. Both imaging methodologies have huge literatures, overviewed for example in (Gardner 1985; Yilmaz 2001).

\section{Imaging and Semblance Conditions}

The phrase "imaging condition" has come to signify a relation between the image volume and the image. I will use the phrase "semblance condition" for another, related concept, namely a quality possessed by the image volume when 
velocity is chosen correctly, and which expresses kinematic compatibility between data and velocity model. The first of these concepts provides a mechanism for producing an image of the subsurface, given a suitable velocity model; the second can lead to analysis and correction of velocity defects.

For the two versions of image volume introduced above, the relation with the image, i.e. with the output of the adjoint operator $D F[v]^{*}$, is clear from comparison of (9) and (10) with (8). For the surface oriented offset image volume, one obtains the image by integrating over h ("stacking"):

$$
I(\mathbf{x})=\int d \mathbf{h} I_{\mathrm{CO}}(\mathbf{x}, \mathbf{h}) .
$$

For the depth oriented offset image volume, on the other hand, one simply sets $\mathbf{h}=0$ ("extract the zero offset section"), which causes (10) to become identical to (8):

$$
I(\mathbf{x})=I_{\mathrm{SS}}(\mathbf{x}, \mathbf{0}) .
$$

I have used "semblance" to describe the nature of the image volume for a data-consistent velocity, motivated by the well-established use of this word in to describe functions which express this consistency or coherence (Taner \& Koehler 1969; Neidell \& Taner 1971). The semblance condition can be expressed as the requirement that the image volume be an eigenvector of the mapping from image volume to image (defined by the imaging condition), with the largest possible eigenvalue.

For surface oriented offset, this means that $I(\mathbf{x}, \mathbf{h})$ should be constant in offset $\mathbf{h}$. In practice, since amplitudes are not controlled in this process (and because the Earth does not conform to the constant density acoustic model), constant phase is an acceptable substitute. The conventional tool with which to judge this quality is the image gather, a $2 \mathrm{D}$ display in which the horizontal image coordinates are fixed and the image amplitudes displayed as function of depth and offset (or shot, or scattering angle,...). If depth is the vertical coordinate in such a plot, constancy (of phase) of $I_{\mathrm{CO}}(\mathbf{x}, \mathbf{h})$ in $\mathbf{h}$ implies that its appearance should resemble horizontal stripes: the gather should be flattened. This criterion is a generalization of the conventional quality assurance metric of standard stacking velocity analysis (Yilmaz 2001). An equivalent quality is strength of the stack, ie. final image: it is strongest when all interference in the sum over $\mathbf{h}$ is constructive (Taner \& Koehler 1969).

Depth oriented offset volumes should on the other hand be focused at zero offset when the velocity is compatible with the data: that is, almost all energy in the volume should be concentrated near $\mathbf{h}=0$. The scattering angle variant should produce flat depth vs. angle gathers.

\section{Velocity Analysis}

Since the image volume constructed by prestack migration should satisfy one of the conditions mentioned in the preceding paragraphs provided that the velocity is consistent with the data, the failure of these conditions to be satisfied provides both evidence that the velocity is not consistent with the data and means to update it to improve its data-consistency. For the surface oriented offset volume, the well-known "smiles and frowns" rule from standard processing velocity analysis carries over almost unaltered, provided that the lateral heterogeneity of the structure to be imaged is not too great:

If an event in an image gather are convex upwards (downwards), then the average velocity above the depth of the event is too high (low).

Similar rules may be devised for updating velocity from depth oriented offset image volumes (Claerbout 1985).

Such rules form the basis for many velocity model building techniques (Lafond \& Levander 1993; Liu \& Bleistein 1995). Some of these techniques are nowadays almost fully automated, and applied to build 3D models. The methods generally require explicit identification of events (picking), thus intrinsically use an intermediate, reduced data volume of picks. Later in this paper I will review attempts to construct model updates directly from the image volume, without event picking.

At this point, the significance of kinematic artifacts in surface oriented image volumes is clear. These artifacts do not conform to the semblance condition for image volumes produced from correct velocities, even for synthetic data. That is, when the velocity structure is sufficiently complex that multipathing is important, image gathers are not in general flat even for correct velocity models (Nolan \& Symes 1997; Brandsberg-Dahl et al. 2003; Stolk \& Symes 2004). Even worse, nothing in the appearance of the artifact events distinguishes them in any obvious way from the actual reflector images (Nolan \& Symes 1997). Thus the appearance of kinematic artifacts in surface oriented image volumes may complicate their use for velocity updating in complex Earth structure.

On the other hand, the landmark work of de Hoop \& Stolk $(2005,2006)$ established that no such artifacts are present in depth oriented (survey-sinking) image volumes, even in the presence of considerable multipathing, under the sole restriction that rays carrying significant energy do not turn horizontal. One might expect that the depth-oriented image volume would exhibit more fidelity to the model, in that every point in the volume depends on all of the data, rather than on a subset (see equation 10). Thus all dips in the data are controlled, and the rays contributing to a given image point are uniquely determined. With some technical caveats, this heuristic reasoning is correct. The result goes a long way towards explaining the widely reported superiority of "wave equation" migration for prestack imaging in regions of complex velocity structure. Note that the use of the wave equation, in the common depth extrapolation depth extrapolation computation of $I_{S S}$, has nothing to do per se with the artifact-free nature of the image volumes constructed by this style of migration - the same results could be obtained with a suitable computational asymptotic representation of the integral formula (10). The restriction to vertically propagating energy may be important however, see (Symes 2002).

\section{EXTENDED MODELING: A UNIFYING CONCEPT}

The extension concept links the image volume constructions of the last section with modeling and inversion: the imaging operators exemplified by the formulae (9) and (10) are in fact adjoints of linearized (Born) extended modeling. 
An extension of model $F: M \rightarrow D$ consists of

- an extended model space $\bar{M}$;

- an extension operator $E: M \rightarrow \bar{M}$;

- an extended modeling operator $\bar{F}: \bar{M} \rightarrow D$ satisfying $F[m]=\bar{F}[E[m]]$ for any $m \in M$.

The extension operator $E$ should be injective, i.e. one-toone, hence enable one to view the model space $M$ as a subset $E[M] \subset \bar{M}$ of the extended model space. Since the extended models will be in some sense unphysical, I will refer to $E[M]$ as the "physical models".

For an extended model as above, the extended inverse problem is: given $d \in D$, find $\bar{m} \in \bar{M}$ so that $\bar{F}[\bar{m}] \simeq d$. Since extended model space has more degrees of freedom, ambiguity is more likely. A solution $\bar{m}$ is physically meaningful only if it is physical, i.e. $\bar{m}=E[m]$. In that case, $m$ is a solution of the original inverse problem, i.e. $F[m] \simeq d$, because of the extension relation between $F$ and $\bar{F}$. That is,

To solve the inverse problem (approximately), find a solution of an extended inverse problem that lies in the range of the extension map.

Application of this concept to migration velocity analysis is based on linearized (tangent) extended modeling. Given an extension as above, its tangent extension consists of

- the tangent extended model space $T \bar{M}$ of pairs $\left(\bar{m}_{0}, \delta \bar{m}\right) \in \bar{M} \times \bar{M}$

- $E$ is typically linear, so its linearization $D E: T M \mapsto \bar{M}$ is defined by $D E\left[m_{0}\right] \delta m=E[\delta m]$.

- linearized (Born) extended modeling: $D \bar{F}: T \bar{M} \rightarrow D$.

From the chain rule, it follows that $D \bar{F}$ is an extension of $D F$, i.e. $D \bar{F}\left[E\left[m_{0}\right]\right] E[\delta m]=D F[m] \delta m$.

It turns out to be reasonable to restrict tangent extensions to perturbations of physical models, i.e. to assume always that $\bar{m}_{0}=E\left[m_{0}\right]$. I shall assume this restriction implicitly in the following discussion, and confound reference models $m_{0}$ with their images $E\left[m_{0}\right]$ under the extension map.

An extension is approximately (linearly) invertible on the data space $D$ if its pseudoinverse is an approximate right inverse:

$$
D \bar{F}\left[m_{0}\right] D \bar{F}\left[m_{0}\right]^{\dagger} d \simeq d
$$

for all $d \in D$. This property is equivalent to approximate surjectivity of the tangent extended operator: that is, for any $d \in D$ and admissible $m_{0}$, one can find an extended model perturbation $\delta \bar{m}$ so that $D \bar{F}\left[m_{0}\right] \delta \bar{m} \simeq d-\bar{F}\left[m_{0}\right]$.

The definition of the data space $D$ may need to be adjusted to satisfy this surjectivity requirement. In the acoustic example, event dips must be limited to those which correspond to non-evanescent waves at the surface. Dip-limiting can be built into the definition of the data space, for example by redefining the norm in $D$ to heavily weight evanescent wave data. Such weighting is straightforward if the admissible class of models includes constraints on wave velocities near the surface

The basic modeling operators considered in this paper are generally not (approximately) surjective. In particular, the linearized modeling operator of $D F[v]$ of the canonical acoustic problem is not surjective in general, unless the data is consistent kinematically with the velocity model $v$. This occurs generically only for very special acquisition geometries, for example single shot or offset gathers. In general a poorly chosen velocity prevents accurate fitting of multioffset data. Put another way, the trivial extension, defined by $\bar{M}=M, E=I, \bar{F}=F$, is almost never invertible for acoustic modeling and its generalizations.

\section{Surface Oriented Offset Extension}

Take for the extended model space $\bar{M}_{\text {SO }}$ a set of positive functions $\bar{v}(\mathbf{x}, \mathbf{h})$ of position $\mathbf{x}$ and offset $\mathbf{h}$. The extension map $E_{\mathrm{SO}}$ simply views a physical velocity (positive function of $\mathbf{x}$ ) as a function of $\mathbf{x}$ and $\mathbf{h}$, i.e. as constant in $\mathbf{h}$ : $E_{\mathrm{SO}}[v](\mathbf{x}, \mathbf{h})=v(\mathbf{x})$.

The extended modeling operator $\bar{F}_{\text {SO }}$ computes the traces for offset $\mathbf{h}$ by using the extended velocity model for offset h, i.e. by solving (2) with $v=\bar{v}(\cdot, \mathbf{h})$.

Tangent extended modeling is similar: $D \bar{F}_{\text {SO }}$ computes the perturbations of all traces with offset $\mathbf{h}$ by solving (4) with $\delta v=\delta \bar{v}(\cdot, \mathbf{h})$. Consistent with the remark above, only physically consistent reference velocities $\bar{v}(\mathbf{x}, \mathbf{h})=v(\mathbf{x})$ will be considered. Accordingly, the tangent extended modeling operator $D \bar{F}_{\text {SO }}$ has an integral representation similar to that of $D F$ :

$$
\begin{gathered}
D \bar{F}_{\mathrm{SO}}[v] \delta \bar{v}\left(t, \mathbf{x}_{s}+2 \mathbf{h} ; \mathbf{x}_{s}\right)=\frac{\partial^{3}}{\partial t^{3}} \int d \mathbf{x} \int d \tau \times \\
G\left(t-\tau, \mathbf{x} ; \mathbf{x}_{s}+2 \mathbf{h}\right) G\left(\tau, \mathbf{x} ; \mathbf{x}_{s}\right) \frac{2 \delta \bar{v}(\mathbf{x}, \mathbf{h})}{v^{3}(\mathbf{x})}
\end{gathered}
$$

which is almost the same as equation (7), in particular $G$ is the same Green's function. The difference is that now the velocity perturbation is allowed to depend on $\mathbf{h}$.

The tangent extended model $D \bar{F}_{\mathrm{SO}}[v]$ appears to be surjective in the sense defined above, i.e. any non-evanescent data can be well approximated by its range for reasonable definitions of the admissible model set. Some theoretical evidence exists for this presumption (Sacks 1988).

The adjoint $D \bar{F}_{\mathrm{SO}}[v]^{*}$ may be read off from the integral expression (11): it is exactly the mapping defined by the integral (9)! That is,

$$
I_{\mathrm{CO}}=D \bar{F}_{\mathrm{SO}}[v]^{*}(d-F[v])
$$

As proven by Beylkin (1985) and Rakesh (1988), in the absence of multipathing, the linearized extended map $D \bar{F}_{\mathrm{SO}}[v]$ is almost unitary: its adjoint differs from its inverse only by dip-dependent scaling and filtering. Thus the common offset image volume is actually seen to be an approximate tangent extended inversion.

The adjoint of $E_{\mathrm{SO}}$ is integration over $\mathbf{h}$, i.e. stacking, so one recovers the relation between image and image volume from the chain rule and the composition relation $F=\bar{F}_{\mathrm{SO}}\left[E_{\mathrm{SO}}\right]$. That is, the imaging condition is simply application of $E_{\mathrm{SO}}^{*}$ :

$$
I=E_{\mathrm{SO}}^{*} I_{\mathrm{CO}}
$$

The other aspect of the imaging condition - the quality of the image volume which signifies data-consistent velocity is also inherent in the extension structure: it is simply membership in the range of $E_{\mathrm{SO}}$. This range consists precisely of extended (linearized) models with $\delta \bar{v}$ independent of $\mathbf{h}$. As 
noted above, for reasons of modeling inaccuracy, use of the adjoint of $D \bar{F}_{\mathrm{SO}}[v]^{*}$ rather than the (pseudo)inverse ("true amplitude migration") operator $D \bar{F}_{\mathrm{SO}}[v]^{\dagger}$, aperture deficits, etc. it is usually necessary to relax this requirement, to membership in the larger subspace obtained from the range of $E_{\mathrm{SO}}$ by slowly-varying amplitude scaling and muting.

Note that when multipathing with significant energy occurs, $D \bar{F}_{\mathrm{SO}}[v]$ is no longer almost unitary: reflector energy may move to the locations of kinematic artifacts, so the adjoint operator is not an approximate inversion up to dip-dependent scaling and filtering (Nolan \& Symes 1997; Brandsberg-Dahl et al. 2003; Stolk \& Symes 2004). Otherwise put, the normal operator is not pseudodifferential, the economical construction of approximate inverses pioneered by Beylkin (1985) is no longer possible, and prestack depth migration does not produce image volumes remotely close to the range of $E$, even when the velocity is data-consistent. This defect is not limited to offset extensions, but also affects those based on other surface acquisition parameters, even scattering angle in the surface oriented definition (Stolk \& Symes 2004).

\section{Depth Oriented Offset Extension}

The nonlinear extended model relevant to wave equation migration requires a bit of a technical digression. I will sketch this reasoning here, both because it will figure in the discussion of the next section and because it establishes the clear-cut route between waveform inversion and migration velocity analysis for the "wave equation" approach.

Denote by $\bar{M}_{\mathrm{DO}}$ the bounded positive selfadjoint operators on $L^{2}\left(\mathbf{R}^{3}\right)$, the Hilbert space of square-integrable functions. Given $\bar{v} \in \bar{M}_{\mathrm{DO}}$, the corresponding generalized acoustic potential field $\bar{u}\left(t, \mathbf{x} ; \mathbf{x}_{s}\right)$ with point source at $\mathbf{x}_{s}$ and wavelet $w(t)$ satisfies

$$
\left(\bar{v}^{-2} \frac{\partial^{2}}{\partial t^{2}}-\nabla^{2}\right) \bar{u}\left(t, \mathbf{x} ; \mathbf{x}_{s}\right)=w(t) \delta\left(\mathbf{x}-\mathbf{x}_{s}\right)
$$

The extended modeling operator is defined by

$$
\bar{F}_{\mathrm{DO}}[\bar{v}]\left(\mathbf{x}_{r}, t ; \mathbf{x}_{s}\right)=\bar{u}\left(t, \mathbf{x}_{r} ; \mathbf{x}_{s}\right)
$$

Define the extension map $E_{\mathrm{DO}}: M \rightarrow \bar{M}_{\mathrm{DO}}$ by

$$
E_{\mathrm{DO}}[v] f(\mathbf{x})=v(\mathbf{x}) f(\mathbf{x}), f \in L^{2}\left(\mathbf{R}^{3}\right)
$$

$E_{\mathrm{DO}}$ is continuous, eg. with the $L^{\infty}$ norm in domain and the operator norm in the range.

Evidently $\bar{F}_{\mathrm{DO}}\left[E_{\mathrm{DO}}\right]=F$, so the foregoing actually does define an extension of $F$.

Acoustic wave velocity is a combination of density and bulk modulus; the bulk modulus gives the volumetric strain response of the material to dilatational stress. Continuum mechanics mandates that this response be local, i.e. intermediated by a diagonal (in space) operator. The extension proposed here models action at a distance: the bulk modulus is represented by a general symmetric positive definite operator, not necessarily diagonal, so that the strain response is not confined to the location of the imposed stress. Physical models, i.e. those in the range of $E_{\mathrm{DO}}$, do not permit action at a distance, hence are modeled by diagonal operators (multiplication by functions). [I am indebted to Scott Morton (personal communication) for this interpretation of the depth-oriented model extension.]
The problem defined in (12) is of course a bit unusual, as the coefficients in the wave equation have become operators, rather than scalars. This problem is mathematically well-behaved, however, with unique finite energy solutions for finite energy wavelet $w(t)$, as follows from slight generalizations of arguments given in Chapter 2 of Stolk's thesis (Stolk 2000a).

The tangent extended modeling operator $D \bar{F}_{\mathrm{DO}}$ is given by

$$
\left.D \bar{F}_{\mathrm{DO}}[v]\right] \delta \bar{v}\left(t, \mathbf{x}_{r} ; \mathbf{x}_{s}\right)=\delta \bar{u}\left(t, \mathbf{x}_{r} ; \mathbf{x}_{s}\right)
$$

where

$$
\left(v^{-2} \frac{\partial^{2}}{\partial t^{2}}-\nabla^{2}\right) \delta \bar{u}\left(t, \mathbf{x} ; \mathbf{x}_{s}\right)=2 v^{-1} \delta \bar{v}\left[v^{-2} \frac{\partial^{2} u}{\partial t^{2}}\right]\left(t, \mathbf{x} ; \mathbf{x}_{s}\right) .
$$

Presume as in the preceding section that the source is impulsive, so that $u=G$. Since we are perturbing only about physical models, the operator on the LHS is the ordinary wave operator. Write the action of the operator $\delta \bar{v}$ as if it were an integral operator, with kernel also denoted $\delta \bar{v}$. Then we can express the solution of (13) as

$$
\begin{gathered}
\delta \bar{u}\left(t, \mathbf{x}_{r} ; \mathbf{x}_{s}\right)=\int d \mathbf{x} \int d \tau G\left(\tau, \mathbf{x} ; \mathbf{x}_{r}\right) \frac{2}{v(\mathbf{x})} \int d \mathbf{x}^{\prime} \times \\
\delta \bar{v}\left(\mathbf{x}, \mathbf{x}^{\prime}\right) \frac{1}{v^{2}\left(\mathbf{x}^{\prime}\right)} \frac{\partial^{2} G}{\partial t^{2}}\left(t-\tau, \mathbf{x}^{\prime} ; \mathbf{x}_{s}\right) .
\end{gathered}
$$

Change variables of integration from $\left(\mathbf{x}, \mathbf{x}^{\prime}\right)$ to $\mathbf{h} \leftarrow\left(\mathbf{x}^{\prime}-\right.$ $\mathbf{x}) / 2$ and $\mathbf{x} \leftarrow\left(\mathbf{x}^{\prime}+\mathbf{x}\right) / 2$. Then the above integral is equal to

$$
\begin{gathered}
=\frac{\partial^{2}}{\partial t^{2}} \int d \mathbf{x} \int d \mathbf{h} \int d \tau \\
G\left(\tau, \mathbf{x}+\mathbf{h} ; \mathbf{x}_{r}\right) G\left(t-\tau, \mathbf{x}-\mathbf{h} ; \mathbf{x}_{s}\right) \frac{2}{v(\mathbf{x}+\mathbf{h}) v^{2}(\mathbf{x}-\mathbf{h})} \delta \bar{v}(\mathbf{x}, \mathbf{h}) .
\end{gathered}
$$

Considerable numerical evidence points to surjectivity of $D \bar{F}_{\mathrm{DO}}[v]$, provided that $v$ is confined to a suitable admissible set and $D$ is defined properly. No theoretical studies of this question have appeared, to the author's knowledge.

The adjoint tangent extended modeling operator is evidently

$$
\begin{gathered}
D \bar{F}_{\mathrm{DO}}[v]^{*}(d-F[v])(\mathbf{x}, \mathbf{h})=\frac{2}{v(\mathbf{x}+\mathbf{h}) v^{2}(\mathbf{x}-\mathbf{h})} \\
\times \int d \mathbf{x}_{s} \int d \mathbf{x}_{r} \int d t \int d \tau G\left(t-\tau, \mathbf{x}+\mathbf{h} ; \mathbf{x}_{r}\right) G\left(\tau, \mathbf{x}-\mathbf{h} ; \mathbf{x}_{s}\right) \times \\
\frac{\partial^{3}(d-F[v])}{\partial t^{3}}\left(t, \mathbf{x}_{r} ; \mathbf{x}_{s}\right),
\end{gathered}
$$

which of course is exactly (10). That is,

the depth oriented image volume (output of survey sinking or "wave equation migration") is the image of the data under the adjoint tangent extended modeling operator, for the depth oriented offset extension:

$$
I_{\mathrm{SS}}=D \bar{F}_{\mathrm{DO}}[v]^{*}(d-F[v]), I=E_{\mathrm{DO}}^{*} I_{\mathrm{SS}} .
$$

As noted above, in a much wider range of cases, including all in which rays carrying significant energy propagate without turning horizontal, the tangent extended operator 
for the depth oriented extension is near-unitary: its pseudoinverse differs from its adjoint by dip-dependent scaling and filterin. Otherwise put, the normal operator for tangent extended depth oriented extended modeling is pseudodifferential, absent turning rays. This variant of prestack depth migration does produce image volumes near the range of the extension map $E$ for data-consistent velocity models, even in the presence of significant multipathing.

\section{OBJECTIVE MIGRATION VELOCITY ANALYSIS}

Migration velocity analysis has a very simple description in the context of tangent extended modeling:

migration velocity analysis $\equiv$ adjust the velocity model $v$ to bring the pseudoinverse solution of the linearized inverse problem $D \bar{F}[v]^{\dagger}(d-F[v])$, or its prestack depth migration approximation $D \bar{F}[v]^{*}(d-F[v])$, as near as possible to the range of the extension map $E$ (the physical models).

The pseudoinverse solution is only computationally feasible when it is closely related (by dip-dependent scaling and filtering) to the prestack depth migration operator. Therefore this procedure really only makes sense for the surface oriented extension when energy moves from data to image volume along essentially unique ray paths. When multiple ray paths carry any significant amount of energy, the depth oriented extension provides a more robust framework for velocity analysis.

Many software tools exist to carry out this task interactively. However it is also possible to view the aim of migration velocity analysis as inversion, and to automate its accomplishment. In fact,

Provided that the underlying extemsion is invertible, migration velocity analysis is an approximate solution method for the partially linearized inverse problem: given data $d$, find velocity model $v$ and short-scale perturbation $\delta v$ so that $D F[v] \delta v \simeq d$ in the least squares sense.

To see this, suppose that migration velocity analysis produces a pseudoinverse solution $D \bar{F}[v]^{\dagger}(d-F[v])$ that lies near the range of $E$, i.e.

$$
D \bar{F}[v]^{\dagger}(d-F[v]) \simeq E \delta v
$$

for a perturbational model $\delta v$. Invertibility of the extension implies that

$$
D F[v] \delta v \simeq D \bar{F}[v] D \bar{F}[v]^{\dagger}(d-F[v]) \simeq d-F[v]
$$

That is, $v$ and $\delta v$ solve the partially linearized inverse problem.

For those cases in which the pseudoinverse is closely related to the adjoint, i.e. in the absence of kinematic artifacts, the criteria for membership in the range of $E$ may be verified by examination of $D \bar{F}[v]^{*}(d-F[v])$ instead, as already noted.

\section{Annihilators}

Since migration velocity analysis actually (implicitly) solves a version of the waveform inversion problem, it is natural to seek objectives whose extrema represent the solution. Since the range of $E$ is a linear subspace of $\bar{M}$, any linear operator vanishing on this subspace gives rise to a quadratic form which can serve as such an objective. A (possibly) $v$ dependent map $A[v]$ from extended model space $\bar{M}$ to some other Hilbert space $H$ is an annihilator of the range of $E$ if

$$
r=E \delta v \Leftrightarrow A[v] r=0
$$

If $A[v]$ is an annihilator in the sense just defined, then the function

$$
J_{A}[v, d] \equiv \frac{1}{2}\left\|A[v] D \bar{F}[v]^{\dagger}(d-F[v])\right\|^{2}
$$

attains its global minimum at velocities which produce physical short-scale models, i.e. image volumes in the range of the extension operator. Any such function could potentially serve as the objective for an optimization approach to migration velocity analysis. Computational advantage can be gained if the pseudoinverse $D \bar{F}[v]^{\dagger}$ can be replaced by the adjoint $D \bar{F}[v]^{*}$ (i.e. prestack depth migration instead of linearized inversion) without seriously perturbing the locations of minimizing models. Such replacement is possible with some choices of $A[v]$.

Of course, the linear space of operators $A[v]$ annihilating the range of $E$ typically has high dimension. Somewhat more surprisingly, the behaviour of the corresponding functions $J_{A}$ varies dramatically with the choice of $A[v]$. The following three types of annihilator have been studied extensively:

- $A[v]=\left(D F[v] D F[v]^{\dagger}-I\right) D \bar{F}[v]$. Under the standing assumption on invertibility of the extension,

$$
\begin{gathered}
J_{A}[v, d]=\frac{1}{2}\left\|\left(D F[v] D F[v]^{\dagger}-I\right) D \bar{F}[v] D \bar{F}[v]^{\dagger}(d-F[v])\right\|^{2} \\
\simeq \frac{1}{2}\left\|D F[v] D F[v]^{\dagger}(d-F[v])-(d-F[v])\right\|^{2} \\
=\min _{\delta v} \frac{1}{2}\|D F[v] \delta v-(d-F[v])\|^{2} .
\end{gathered}
$$

Thus minimizing $J_{A}$ over $v$ is equivalent to output least squares solution of the partially linearized problem. So even output least squares fits in this "annihilator" framework.

- $A=E E^{\dagger}-I$. Note that

$$
\left\|\left(E E^{\dagger}-I\right) r\right\|^{2}=\left\|E E^{\dagger} r\right\|^{2}-2\left\langle r, E E^{\dagger} r\right\rangle+\|r\|^{2} .
$$

Recalling that $E$ may be taken to be an isometry, i.e. $E^{*}=$ $E^{\dagger}$. Using this fact and the pseudoinverse property $E E^{\dagger} E=$ $E$, the last line simplifies to

$$
=\|r\|^{2}-\left\|E^{\dagger} r\right\|^{2}
$$

Thus minimizing $J_{A}$ in this case is the same as maximizing $\left\|E^{\dagger} D \bar{F}[v]^{\dagger}(d-F[v])\right\|^{2}$. Provided that $D \bar{F}[v]$ is almost unitary, same optima occur using prestack depth migration rather than pseudoinversion, i.e. minimizing $J_{A}$ yields the same velocity model as does maximizing $\| E^{*} D \bar{F}[v]^{*}(d-$ $F[v])\left\|^{2}=\right\| D F[v]^{*}(d-F[v]) \|^{2}$. For the surface oriented extension, the resulting functional is the simplest version of (total)stack power, and was studied in the context of convolutional modeling by Toldi (1989). For either type of extension described above, it amounts to the image power (Soubaras \& Gratacos 2006; Shen \& Calandra 2005). 
- $A=\left(1-\nabla_{\mathbf{x}, \mathbf{h}}^{2}\right)^{-\frac{1}{2}} \partial_{\mathbf{h}}$ for the surface oriented extension, and $\mathbf{h}$ (i.e. multiplication by offset) for the depth oriented extension, and various inessential modifications of these operators. Introduced by the author (Symes 1986b), this type of annihilator has come to be called differential semblance.

We have already discussed the behaviour of the output least squares function at some length, and this partially linearized version of it shares the same propensity for multimodality. Prestack depth migration cannot in general be used as a straightforward substitute for linearized inversion in this partially linearized output least squares formulation of objective velocity analysis: the mismatches of amplitudes can actually generate yet more spurious stationary points.

The second choice (image power) is much less affected by the amplitude differences between (pseudo)inversion and prestack depth migration, but also exhibits a pronounced tendency to multimodality: with prototypical data bandwidth and acquisition geometry, many critical points typically appear, most having nothing to do with a physically meaningful solution. Chauris has carefully illustrated this phenomenon, and noted that it is caused by isolated tangencies between unrelated events in predicted and target data (Chauris 2000; Chauris \& Noble 2001). As is the case with output least squares, a sufficiently good initial guess, made easier to supply by downfiltering the data in early stages, can yield convergence for image power optimization. Soubaras has recently provided an excellent example of this strategy (Soubaras \& Gratacos 2006), and it has been used to good effect by Shen and colleagues as well (Shen \& Calandra 2005; Shen et al. 2005a).

The differential semblance class (third option from list above) has many variants, some employing prestack depth migration (i.e. $D \bar{F}[v]^{*}$ ) rather than (pseudo)inversion to construct the function $J_{A}$. Differential semblance variants based on surface oriented extension have used

- convolutional model simulation of plane wave data (Symes 1986b, 1990; Symes \& Carazzone 1991; Symes 1991b; Symes \& Carazzone 1992; Minkoff \& Symes 1997),

- convolutional model simulation of CMP data (Symes 1993; Symes \& Gockenbach 1995; Symes 1998; Li \& Symes 2005; Dussaud \& Symes 2005; Verm \& Symes 2006),

- two-way wave equation modeling (two-way reverse time migration) (Symes 1991c; Symes \& Versteeg 1993; Kern \& Symes 1994),

- generalized Radon transform simulation of acoustic scattering (Kirchhoff migration) (Chauris \& Noble 2001; Mulder \& ten Kroode 2002; de Hoop et al. 2003), and

- generalized Radon transform simulation of anisotropic elastic scattering (de Hoop et al. 2005).

Variants based on depth oriented extension have employed

- one-way wave equation migration of shot profiles (Shen et al. 2005b; Albertin et al. 2006), and

- one-way wave equation migration via the DSR equation (Shen et al. 2003; Khoury et al. 2006).

The same concept has been applied to cross-well tomography (Plessix 2000).

\section{Mathematical Characterization of Differential Semblance}

Differential semblance has a precise mathematical characterization, which partly explains its significance:

Amongst all possible quadratic forms in the data, parametrized by velocity, of the form given in equation (14), only differential semblance (and inessential variations thereof) is smooth jointly as a function of smooth perturbations in velocity and finite energy perturbations in data (Stolk 8 Symes 2003).

More precisely, the annihilator $A$ must be belong to the class of pseudodifferential operators, mentioned earlier in connection with the normal (Hessian) operator of linear scattering. This statement is a paraphrase of the precise mathematical fact; the reader may consult the reference for the "fine print". The significance of this statement lies in the requirement of smoothness under variation of both velocity and finite energy data, i.e. data perturbations of unlimited bandwidth. A related statement is that the shape of the differential semblance objective function is stable as data sampling is refined and/or upper bandlimit increased, and no other form of $J_{A}$ has this property - not image power, not output least squares, nor any other yet-to-be-invented quadratic forms. Since any smooth objective in this sense necessarily has a partial Hessian operator in $d$, of the form given in equation (14), this result also shows that any function of $v, d$ must be identical to differential semblance to second order in $d$, if it is to be smooth in the sense specified.

Pseudodifferential operators share many properties with differential operators: indeed, they form an algebra, generated by differential operators and all powers of the Laplace operator. They are expressed as integral operators whose kernel is a the product of the Fourier sinusoid with a so-called symbol, a function of position and wavenumber. The symbol and its derivatives are required to grow like a homogeneous function of the wavenumber, at large wavenumber, as do polynomials (but so do some non-polynomial functions); differential operators form a special subalgebra, for which the symbol is a polynomial in wavenumber. It is a remarkable fact that annihilators generating smooth objectives for migration velocity analysis must belong to this very special class of operators.

The argument given in (Stolk \& Symes 2003) is quite technical; it generaizes a characterization of pseudodifferential operators due to Cordes (Taylor (1981), Ch. VIII). The essence however is fairly straightforward, and a simple one-dimensional model problem will give the reader some inkling. Observe that migration involves moving and stretching of events. The linear operator of composition with affine motion of the real line is thus a simple ana$\log$ of migration. The analog of the question answered in (Stolk \& Symes 2003) is: for which bounded operators $A$ on square-integrable functions is the quadratic form, generated by composing the operator with affine motions, smooth as a function of the affine motion parameters and the input square-integrable function?

Affine motions of the line take the form $t \mapsto \tau+\alpha t$, and are parametrized by a shift $\tau$ and a dilation factor $\alpha$ Composition of a function $u(t)$ with an affine motion of shift by $\tau$ and stretch or dilation by the factor $\alpha$ yields the operator $T_{\tau, \alpha} u(t)=u(\tau+\alpha t)$.

Given a bounded operator $A$ on the Hilbert space 
of square-integrable functions on the real line, define the quadratic form in $J_{A}[\tau, \alpha ; u]$, parametrized by $\tau, \alpha$, by analogy with the construction explained in the preceding subsection:

$$
J_{A}[\tau, \alpha ; u]=\frac{1}{2} \int d t\left|\left(A T_{\tau, \alpha} u\right)(t)\right|^{2}
$$

For convenience I will assume that $A$ is represented by an integral with kernel $a$ :

$$
A u(t)=\int d s a(t, s) u(s)
$$

and that $a(t, s)$ vanishes for large $|t|,|s|$. This assumption is not essential, and can be removed at the price of a more sophisticated and less transparent argument.

Smoothness of the quadratic forms mentioned in the preceding subsection is analogous to smoothness of

$$
(\tau, \alpha, u) \mapsto J_{A}[\tau, \alpha ; u] .
$$

If the gradient (in $u$ ) of $J_{A}$ is to be smooth as well (as is required for reliable convergence of Newton's method), then the mappings

$$
(\tau, u) \mapsto T_{\tau, 0}^{*} B T_{\tau, 0} u,(\alpha, u) \mapsto T_{0, \alpha}^{*} B T_{0, \alpha} u
$$

must also be differentiable; here $B=A^{*} A$ is also an integral operator, with kernel related to that of $A$. Since the kernel of $A$ is assumed to vanish outside a square, it follows that the kernel $b(t, s)$ of $B$ vanishes outside a (possiblly bigger) square. Assume that $b$ is also regular enough to have a welldefined Fourier transform $p(t, \omega)$ in its second argument, i.e.

$$
p(t, \omega)=\int d s e^{-i s \omega} b(t, s) .
$$

Then by Plancherel's Theorem,

$$
B u(t)=\frac{1}{2 \pi} \int d \omega p(t, \omega) e^{i \omega t} \hat{u}(\omega)
$$

in which $\hat{u}$ is the Fourier transform of $u$. Thus $p$ plays the role of a symbol, and $B$ is a pseudodifferential operator if $p$ grows like a homogeneous function of $|\omega|$ for large $\omega$. It is straightforward to see that

$$
\begin{gathered}
\left.\frac{\partial}{\partial \tau} T_{\tau, 0}^{*} B T_{\tau, 0} u\right|_{\tau=0}=\frac{1}{2 \pi} \int d \omega \frac{\partial p}{\partial t}(t, \omega) e^{i \omega t} \hat{u}(\omega) \\
\left.\frac{\partial}{\partial \alpha} T_{0, \alpha}^{*} B T_{0, \alpha} u\right|_{\tau=0}=\frac{1}{2 \pi} \int d \omega\left(\omega \frac{\partial p}{\partial \omega}-t \frac{\partial p}{\partial t}\right)(t, \omega) e^{i \omega t} \hat{u}(\omega) .
\end{gathered}
$$

Since the support of $b$ is bounded, the choice $u(t)=e^{-i \omega_{0} t}$ is effectively square-integrable (set to zero where $b=0$ ), so that for a constant $K$ independent of $\omega_{0}$ and $t$,

$$
\left|\frac{\partial p}{\partial t}\left(t, \omega_{0}\right)\right| \leq K,\left|\omega \frac{\partial p}{\partial \omega}\left(t, \omega_{0}\right)\right| \leq K .
$$

Iterating this reasoning and rearranging, conclude that for any $k, m \geq 0$,

$$
\left|\frac{\partial^{k}}{\partial t^{k}} \frac{\partial^{m}}{\partial \omega^{m}} p(t, \omega)\right| \leq K_{k, m}(1+|\omega|)^{-m}
$$

for a sequence of constants $K_{k, m}$ independent of $t$ and $\omega$. This estimate says precisely that the symbol $p$, together with all of its derivatives, grows like a homogenous function (of order zero) as $|\omega| \rightarrow \infty$, i.e. has the property required to conclude that $B$ is a pseudodifferential operator.
This argument does not show that $A$ is pseudodifferential. However symmetric nonnegative definite pseudodifferential operators like $B$ have pseudodifferential square roots. Replacing $A$ with $B^{\frac{1}{2}}$ does not change the quadratic form $J_{A}$, whence $A$ might as well be pseudodifferential.

Technically more involved arguments are required to replace affine motions with the prestack migration operator $D \bar{F}[v]$ and to conclude that annihilators $A$ generating smooth (in $v, d$ ) quadratic forms $J_{A}[v, d]$ for migration velocity analysis must be pseudodifferential, but the essential concept is the same.

The numerical work cited above suggest in one way or another than not only is the differential semblance objective stable against high-frequency data perturbation, but it is also essentially monomodal: the only stationary points are physically significant solutions of the waveform inversion problem (and, in particular, velocities kinematically consistent with data). In one special case, this has even been proven with mathematical rigor: for the differential semblance variant for CMP data, based on hyperbolic NMO (Symes \& Gockenbach 1995; Symes 1998; Li \& Symes 2005; Verm \& Symes 2006; Li \& Symes 2007), all stationary points are global minima, up to an error proportional to a dominant wavelength (Symes 1999, 2001). The essential idea of the proof is the relation between the differential semblance objective and fitting of apparent velocities in the data, an approach to velocity estimation also known as stereotomography (Billette \& Lambaré 1998). That is, differential semblance is essentially data-weighted stereotomography, implicitly computed without event picking (see also (Chauris 2000; Chauris \& Noble 2001)).

\section{Influence of Coherent Noise}

The linearized description of seismic scattering models single or primary scattering. It neglects multiple scattering, so that any multiply scattered energy in the data appears as coherent noise, with apparent velocity differing from that of the primary events. Since multiple reflections are not a negligible component of typical reflection data, the influence of this signal type on objective migration velocity analysis algorithms must necessarily play a role in determining their practical impact.

The contrasting behaviors of the various migration velocity analysis objectives carry over to their response to the presence of coherent noise such as multiple reflections. As noted above, output least squares and image power optimization via Newton-like algorithms require good initial estimates of velocity structure in order to converge to a physically relevant final velocity estimate. However, output least squares and image power optima are not much influenced by presence of events with differing apparent velocity, which are effectively orthogonal signal components hence thrown into the residual (output least squares) or stacked out (image power). Differential semblance optima, on the other hand, are quite stable against changes in initial estimate but are strongly influenced by coherent noise, tending to yield averages of apparent velocities weighted by the corresponding event strengths (Gockenbach \& Symes 1999; Mulder \& ten Kroode 2002; Li \& Symes 2005; Verm \& Symes 2006).

Several "signal processing" approaches have been sug- 
gested to retain the global convergence properties of differential semblance optimization while reducing its sensitivity to coherent noise. An obvious approach is application of one or more multiple suppression algorithms to the data, to render it more similar to the primaries-only data modeled by the Born approximation (Gockenbach \& Symes 1999; Li \& Symes 2005). Since the tendency of differential semblance is to average the apparent velocities of events, the lower velocities of multiple reflections in near-layered structures will tend to appear undercorrected in image gathers, whereas the primary events appear overcorrected. Therefore, dipfiltering the undercorrected events and remodeling will have the effect of suppressing multiple reflections, under these assumptions (Mulder \& ten Kroode 2002; Li \& Symes 2007). Finally, the extremal regularization algorithm (Gockenbach \& Symes 1999) perturbs data as well as velocity to find the well-fit data closest to given data. In the event that the primary reflections form the strongest consistent family of reflections in the data, the extremal regularization algorithm will find them (Gockenbach \& Symes 1999).

Another approach to desensitization of differential semblance optimization to the presence of coherent noise relies on continuation or homotopy, using the differential semblance velocity estimate as a starting guess, then introducing either a blend of differential semblance and image power or output least squares objectives, or simply switching to image power or output least squares. Gockenbach analyzed a homotopy between differential semblance and output least squares in his thesis, and showed that with some reasonable assumptions one could guarantee that the path beginning at the differential semblance solution terminates as the output least squares solution (Gockenbach et al. 1995). Chauris (2000); Chauris \& Noble (2001) employed a similar approach using image power, as did Shen and collaborators (Shen et al. 2005b; Shen \& Calandra 2005). Celis and Larner proposed a similar homotopy, computing image power or an equivalent crosscorrelation over offset windows of varying width; in the limit of small width, the crosscorrelation is very close to differential semblance in its behviour (Celis \& Larner 2002). All of these homotopies share an implicit constraint on the overall noise energy with extremal regularization: if the noise is sufficiently large, the homotopy begins at a highly corrupted differential semblance estimate and terminates at a spurious stack power peak or output least squares local minimum.

All of these ideas have been shown to be effective in limited circumstances. However, all are somewhat ad hoc. Multiple scattering is a physical phenomenon predicted by modeling, and evident in much - perhaps almost all - field data. This observation suggests development of a different and more fundamental approach to migration velocity analysis, based on full nonlinear modeling. Such an approach would at first appear to suffer from internal contradiction, since migration is tied to linearized modeling. However the extended modeling point of view provides a way to resolve this conceptual conflict.

\section{TOWARDS EFFECTIVE NONLINEAR WAVEFORM INVERSION}

Each of the extensions underlying the two common vari- ants of prestack depth migration is the linearization of a full waveform modeling extension. In all cases examined in this paper, the extension map $E$ is linear, so linear annihilators $A$ may be used to characterize its range and hence the physically significant solutions of the extended nonlinear inverse problem.

Accordingly, suppose that $\bar{F}: \bar{M} \rightarrow D$ and $E: M \rightarrow$ $\bar{M}$ form an extension of $F: M \rightarrow D$, and that $A$ is an annihilator of the range of $E$. For the present discussion, in contrast to that of the last section and without compelling reason to do otherwise, suppose that $A$ is a fixed, linear map. Given $d \in D$ and a fit tolerance $\epsilon>0$, define the feasible model set $S_{\epsilon}[d]$ by

$$
S_{\epsilon}[d]=\{\bar{m} \in \bar{M}:\|\bar{F}[\bar{m}]-d\| \leq \epsilon\|d\|\},
$$

and $J_{A}: \bar{M} \rightarrow \mathbf{R}$ by

$$
J_{A}[\bar{m}]=\frac{1}{2}\|A \bar{m}\|^{2} .
$$

A solution of the constrained optimization problem

$$
\text { minimize } J_{A}[\bar{m}] \text { subject to } \bar{m} \in S_{\epsilon}[d]
$$

is then a candidate for solution of the waveform inversion problem. If the objective value is near zero, then the solution $\bar{m}$ predicts the data to within the specified tolerance, and is close to the range of $E$ in the sense that its image under $A$ is small. Therefore there exists a model $m \in M$ with $E m \simeq \bar{m}$, hence $F[m] \simeq d$. Conversely, if the data $d \in D$ is consistent with the model to tolerance $\epsilon$, that is, if $m \in M$ exists for which $\|F[m]-d\|<\epsilon$, then $\bar{m}=E m$ is a priori a solution of problem (15). Of course the constrained optimization problem (15) can be rewritten as an unconstrained problem by use of a penalty (Lagrange multiplier) formulation.

In general, two major issues arise in formulating any approach to the solution of problem (15):

- representation of the extended model $\bar{M}$, and

- parametrization of the feasible model set $S_{\epsilon}[d]$.

The first issue presents few difficulties for some surfaceoriented extensions, a circumstance evident in the theory developed in (Symes 1991a). The extended model simply amounts to permitting the coefficients in the wave equation to depend on a surface acquisition parameter, and simulation is independent for each value of the parameter. Thus the computational complexity of the extended modeling operator is no greater than that of the basic modeling operator. The memory complexity is equivalent to that of image volume formation in prestack depth migration. [Common offset modeling is an exception to this rule - it does not appear to be possible to model common offset data gathers without dramatically increased computational effort, essentially the same effort as modeling each trace independently.]

For depth oriented extensions, the coefficients in the wave equation (vectors in the extended model space) are positive definite symmetric operators. The kernels or matrices of these operators occupy storage equivalent to that of image volumes in prestack depth migration. However the computational complexity of modeling via timestepping for generalized wave equations such as (12) is potentially enormous, apparently involving a matrix multiplication at each 
time step. In special cases, such as the layered model discussed below, the cost of modeling can be reduced dramatically by judicious choice of basis in model space. I will come back to this issue, which is currently very much open, in the concluding discussion.

The force of the second issue arises from the very irregular geometry of the set $S_{\epsilon}[d]$. [For an explicit discussion of a closely related phenomenon and a careful quantification, see (Chavent 1991).] This puts the constrained optimization problem (15), in its original form, beyond the reach of sequential quadratic programming, which amounts to Newton's method applied to the first order necessary conditions for a constrained optimum (Nocedal \& Wright 1999). As for all other versions of Newton's method, smoothness of the objective (in this case, the Lagrangian function) is essential. However the Lagrangian is just as irregular as the output least squares objective discussed earlier. A reparametrization is essential, so that the problem becomes a smooth one amenable to relatives of Newton's method.

Note that migration velocity analysis, viewed as a route to partially linearized inversion via tangent extended modeling, could be formulated in the same way, with the same difficulty. A reparametrization of the feasible set is built into the formulation of the problem: the analogue of $S_{\epsilon}$ for migration velocity analysis consists of the set of pairs $v, D \bar{F}[v]^{\dagger} d$, parametrized by the macromodel velocity $v$ and the data $d$. That is, for migration velocity analysis, the velocity model naturally parametrizes the feasible set. This set is also highly curved, but the evaluation of $J_{A}$ (as defined in this section) on this set, viewed as a function of $v$, is smooth provided that an appropriate choice of $A$ is made, as noted above.

An effective reparametrization of $S_{\epsilon}[d]$ for layered modeling uses the well-known slowness-dependent traveltime parametrization of depth. The author studied a penalty reformulation of (15) for plane wave solutions of a layered acoustic model with surface-oriented extension in (Symes 1991a). I used traveltime parametrization and a natural version of differential semblance for $A$. I showed that the Hessian of the penalty function at a consistent solution is positive definite, which implies the uniqueness of the stationary point at least locally, i.e. absence of spurious stationary points. To obtain positivity of the Hessian, I imposed a scale dichotomy property on the model, which is reminiscent of the scale dichotomy used to justify use of perturbation theory. This study did not address the determination of appropriate values for the penalty parameter, an essential step in any practical implementation.

Parametrization by traveltime is not available in general for multidimensional problems. However one can equally well view this step as parametrization of the model by the data together with the long-scale part of the velocity model, which of course determines the relation between traveltime and depth. In the linearized multidimensional setting, this concept was the foundation of the Model-Based Traveltime approach to migration velocity analysis proposed by Chavent and his co-workers (Clément \& Chavent 1993; Plessix et al. 1995).

A drawback to parametrizing the feasible set by the long-scale part of the velocity is the very definition of "longscale": the term refers implicitly to spatial wavelength, which is model-dependent. An alternative choice would be the low-frequency band not supplied in field data, via the solvability of the impulsive inverse problem (i.e. with data at all frequencies including $0 \mathrm{~Hz}$ ). This is the approach actually taken in the layered medium study mentioned above (Symes 1991a). Some numerical evidence (but little theoretical backup) exists that the impulsive several dimensional nonlinear problem also has unique solutions. For example, the previously mentioned work of Bunks, Chavent, and collaborators (Bunks et al. 1995), also that of Shin and collaborators (Shin \& Min 2006), may be interpreted as suggesting that the $2 \mathrm{D}$ common source point inverse problem has a well-defined and computationally tractable solution provided that essentially impulsive data is available. This evidence supports the hypothesis that solution of impulsive source problems may provide appropriate reparametrization for $S_{\epsilon}[d]$, at least for surface oriented extension.

No evidence, numerical or otherwise, seems to exist concerning constructive reparametrization of the depth oriented extension in its nonlinear form. It is possible however to analyze a special case, that of layered models.

\section{Depth oriented extension for layered models}

Assume that the Earth is horizontally layered, and that the depth oriented extended model (12) should also model laterally homogeneous action-at-a-distance. That is, shifting the source location $\mathbf{x}_{s}$ horizontally should produce a corresponding shift in the wavefield $\bar{u}$ in the extended model (12). Assuming that the source and receiver positions lie at the same depth, and that the offsets are the same from shot to shot, it follows that the data is also a function of offset only. Moreover, it follows that the operator $\bar{v}^{-2}$ is necessarily a convolution operator in the horizontal variables, For computational purposes, it is convenient to represent $\bar{v}^{2}$ as convolution with a kernel $\sigma$, and for convenience I will assume a 2D Earth (or equally well a line source in the $y$ direction). For any function $g(z, x)$ even in $x$,

$$
\bar{v}^{2} g(z, x)=\frac{1}{\pi} \int d k \cos k x \hat{\sigma}(z . k) \hat{g}(z, k),
$$

where $\hat{g}$ is the cosine transform of $g$. Assume, also for convenience, that the velocity field is known near the surface, and in particular at the source depth, and is physical: for $z$ near $z_{s}, \bar{v}(z, x)=v_{0} \delta\left(z-z_{s}\right) \delta\left(x-x_{s}\right)$. Locating the source for convenience at $x_{s}=0$, the wavefield $u$ is perforce even in $\mathrm{x}$. The extended modeling equation (12) then implies for the cosine transform $\hat{u}$

$$
\left[\frac{\partial^{2}}{\partial t^{2}}-\hat{\sigma}(z, k)\left(\frac{\partial^{2}}{\partial z^{2}}-k^{2}\right)\right] \hat{u}(t, z, k)=v_{0}^{2} w(t) \delta\left(z-z_{s}\right) .
$$

Representing the operator $\bar{v}^{2}$ in this way has made its action sparse (diagonal) in an appropriate basis, so that its computational cost is reduced to the same level as that of a physical velocity model (i.e. multiplication operator). Accordingly, the wave equation (16) may be solved as efficiently as the basic modeling equation (2) (actually, more efficiently, in this case).

The extended forward modeling operator has the representation

$$
\bar{F}[\bar{v}]\left(t, z_{r}, x_{r}\right)=\frac{1}{\pi} \int d k \cos k x_{r} \frac{\partial \hat{u}}{\partial t}\left(t, z_{r}, k\right)
$$

in terms of the cosine transformed field $\hat{u}$, which suggests an efficient implementation of $\bar{F}$. 
A simple finite difference scheme of order 2 in time and 4 in space is amply accurate to approximate the solution of the extended modeling equation (16). Discrete cosine transform then provides a natural and efficient implementation of the extended modeling operator via (17). To obtain points in the feasible model set $S_{\epsilon}[d]$, I approximated the solution of the extended inversion problem

$$
\text { minimize }\|\bar{F}[\bar{v}]-d\| \text { over } \bar{v}
$$

using a quasi-Newton method (limited memory BroydenFletcher-Goldfarb-Shanno, see (Nocedal \& Wright 1999)). This technique required computation of the gradient of the objective appearing in (18). I computed the gradient using the adjoint state method (Tarantola 1987; Plessix 2006). A useful by-product of these constructions was a quasi-Newton method for solving the output least squares problem (1) for the basic forward modeling operator $F$ of layered acoustics.

The examples to follow all pertain to the same simple three-layer model, depicted in Figure 1. The surface $z=0$ of this model is pressure-free, modeled by a Dirichlet boundary condition. The reflectivities are chosen so that the free surface multiple reflection from the first layer is roughly the same strength as the primary reflection from the second layer, see Figure 2.

Iterative output least squares inversion for a layered velocity model yields results which depend strongly on the initial estimate, consistent with the accumulated experience of other studies as reviewed above. Three such results are depicted in Figure 3. Only the inversion from the initial model with correct velocity in the second layer (blue curves in Figure 3) places the second reflector at the right depth. Inversion cannot of course correct the velocity below the second interface, as no reflections return from deeper in the model. Each iteration was halted when the gradient decreased in length by two orders of magnitude from its initial value.

Iterative extended output least squares inversion also produced results depending on the initial estimate. For the same initial estimates as in used in the output least squares trials (Figure 3), or rather their extensions (images under $E)$, the extended inversions are depicted in Figures 4, 5, and 6 . In all three cases, Broyden-Fletcher-Goldfarb-Shanno iteration was terminated when the RMS fit error declined to $1 \%$. The predicted seismograms, that is, $\bar{F}[\bar{v}]$, are shown for the three cases in Figures 7, 8, and 9. All are visually indistinguishable from the data depicted in Figure 2.

These results suggest several conjectures about this particular extended inverse problem:

- The range of $\bar{F}$ appears to be stable (analogue of surjectivity for linearized extention), and fairly precise fits to data are possible via local optimization (limited memory Broyden-Fletcher-Goldfarb-Shanno) starting at a wide variety of initial model estimates;

- Only the extended output least squares estimate produced by a kinematically accurate initial model (Figure 6) is reasonably close to the range of the extension map $E$, i.e. concentrated near zero offset. Moreover, the degree of concentration appears to change smoothly as the initial velocity becomes kinematically more accurate;

- The treatment of the extended output least squares problem could benefit from some regularization (not applied in these experiments): all estimates produced in this series are relatively noisy.
Thus the nonlinear analogue of the surjectivity property important in the migration velocity analysis analysis, appears to hold at least to the limited extent investigated in these examples. Furthermore, the behavior of these extended output least squares inversions is roughly consistent with the expectation that the function $J_{A}$, for $A=$ multiplication by offset, may in some sense change smoothly with position in the feasible model set $S_{\epsilon}[d]$ (in which Figures 4 through 6 represent three points).

\section{DISCUSSION AND CONCLUSION}

The foregoing discussion has reviewed the failure of waveform (output least squares) inversion to update macromodels and the contrasting ability of migration velocity analysis to do so. Migration velocity analysis is based on linearized or Born modeling, and has at least two major variants corresponding to two approaches to image volume formation. The extension concept formalizes image volume formation as application to data of an adjoint (or pseudoinverse) tangent modeling operator. This identification clarifies the meaning of the tendency of surface oriented image volumes to exhibit kinematic artifacts in the presence of multipathing: the corresponding extended adjoint tangent extended modeling operator is not a good approximation to the pseudoinverse. The depth oriented extension on the other hand maintains a close relationship (modulo dip-dependent amplitude scaling and filtering) between adjoint and pseudoinverse tangent extended modeling operators under a much wider range of circumstances. In both cases, migration velocity analysis may be cast as an optimization problem in a number of ways. Only one of these, differential semblance, appears to yield optima that are robust against large errors in initial model estimates.

The extension concept permits a unified view of output least squares and migration velocity analysis approaches to Earth model estimation: migration velocity analysis can be regarded as a solution method for a partially linearized output least squares inverse problem. The extension framework also suggests an approach to nonlinear waveform inversion incorporating elements of migration velocity analysis. Preliminary numerical experiments hint that this nonlinear extension may serve as the basis for model updates incorporating substantial velocity changes and accounting properly for multiple reflections (and, in more general settings, other physics of waves).

Two remaining obstacles must be overcome before this nonlinear migration velocity analysis approach to waveform inversion becomes in any sense practical. The first concerns the depth oriented extension: in this formulation, nonphysical models are operators, and must be represented as sparse matrices in order that timestepping simulation is computationally feasible. The simple solution sketched above for layered models, via convolution and the Fourier transform, is not available in general. A possible resolution may lie in a phase space representation: the kernels of these operators are analogous to space shift image volumes in survey-sinking prestack depth migration, and these data tend to be very sparse in phase space (one or at most a few dips at any one point in space). Fast algorithms have been developed in the last few years for representation of functions possessing 
the typical phase space sparsity properties of seismic images (Candes \& Demanet 2005; Chauris 2006). The operator coefficients appearing in the nonlinear depth oriented extension thus might be expected to be sparse in phase space as well, that is, to have a sparse matrix representation in terms of curvelets or another multiresolution frame.

The second obstacle is the problem of extreme geometric irregularity of the feasible model set $S_{\epsilon}[d]$, and the consequent poor performance of standard constrained optimization tools such as sequential quadratic programming, which typically fail to solve the problem (15) when applied directly. Earlier work on the layered acoustic problem (Symes 1991a) suggested that reparametrizing the feasible model set via impulse response inversion (that is, viewing the missing low frequency components as parameters) may circumvent this difficulty. Some numerical evidence indicates that impulse response inversion for surface oriented extensions may indeed be possible, for nonlayered problems as well. Whether a similar statement can be made for depth oriented extensions remains to be seen. This is a matter of some import, as the nonlinear scattering effects which motivate the nonlinear migration velocity analysis approach sketched here tend to occur in conjunction with complex ray geometry. A notorious example of this phenomenon occurs in the vicinity of salt bodies embedded in sedimentary sequences, giving rise to rugose high contrast interfaces generating complex ray paths and strong multiple reflections. In the presence of such complexity (multipathing), the structure of the inverse problem for depth oriented extensions is simpler (more straightforward relation between adjoint and pseudoinverse for tangent operators) than is the case for surface oriented extensions. This reasoning suggests that the impulse response inverse problem for depth oriented extension is worth some study.

\section{ACKNOWLEDGEMENTS}

I am indebted to many colleagues for their insights into velocity analysis and seismic inversion. I thank the Editor and Associate Editor of Geophysical Prospecting for important suggestions, and the three anonymous referees for very careful critiques which led to many improvements in the manuscript. The work reported here was supported in part by the sponsors of The Rice Inversion Project.

\section{REFERENCES}

Albertin, U., Sava, P., Etgen, J., \& Maharramov, M., 2006. Adjoint wave equation velocity analysis, in Expanded Abstracts, p. TOM2.1, Society of Exploration Geophysicists.

Backus, G. \& Gilbert, F., 1968. The resolving power of gross earth data, Geophysical Journal of the Royal Astronomical Society, 16, 169-205.

Backus, G. \& Gilbert, F., 1970. Uniqueness in the inversion of inaccurate gross earth data, Philosophical Transactions of the Royal Society of London, Series A, 266, 123-192.

Bamberger, A., Chavent, G., \& Lailly, P., 1977. Etude mathématique et numérique d'un problem inverse pour l'Équation des ondes a une dimension, Rapport Interne 14, Centre de Mathématiques Appliqués, École Polytechnique, Paris.

Bamberger, A., Chavent, G., \& Lailly, P., 1979. About the stability of the inverse problem in 1-d wave equation - application to the interpretation of seismic profiles, Applied Mathematics and Optimization, 5, 1-47.

Beylkin, G., 1985. Imaging of discontinuities in the inverse scattering problem by inversion of a causal generalized radon transform, Journal of Mathematical Physics, 26, 99-108.

Beylkin, G. \& Burridge, R., 1990. Linearized inverse scattering problem of acoustics and elasticity, Wave Motion, 12, 15-22.

Billette, F. \& Lambaré, G., 1998. Velocity macro-model estimation from seismic reflection data by stereotomography, Geophysical Journal International, 135, 671-680.

Biondi, B., 2003. Equivalence of source-receiver migration and shot-profile migration, Geophysics, 68, 1340-1347.

Biondi, B. \& Palacharla, G., 1996. 3-d prestack migration of common-azimuth data, Geophysics, 61, 1533-1543.

Biondi, B. \& Shan, G., 2002. Prestack imaging of overturned reflections by reverse time migration, in Expanded Abstracts, pp. 1284-1287, Society of Exploration Geophysicists.

Biondi, B. \& Symes, W., 2004. Angle-domain commonimage gathers for migration velocity analysis by wavefieldcontinuation imaging, Geophysics, 69, 1283-1298.

Bleistein, N., 1987. On the imaging of reflectors in the earth, Geophysics, 52, 931-942.

Brandsberg-Dahl, S., de Hoop, M., \& Ursin, B., 2003. Focusing in dip and AVA compensation on scattering angle/azimuth common image gathers, Geophysics, 68, 232-254.

Brenders, A. \& Pratt, G., 2006. Full waveform tomography for lithospheric imaging: results from a blind test in a realistic crustal model, Geophysical Journal International, (in press).

Brenders, A. \& Pratt, G., 2006. Efficient waveform tomography for lithospheric imaging: implications for realistic, 2-d acquisition geometries and low frequency data, Geophysical Journal International, (in press).

Bube, K. \& Burridge, R., 1983. The one dimensional inverse problem of reflection seismology, SIAM Review, 25(4), 497559.

Bunks, C., Saleck, F., Zaleski, S., \& Chavent, G., 1995. Multiscale seismic waveform inversion, Geophysics, 60, 1457-1473.

Burridge, R., de Hoop, M., Miller, D., \& Spencer, C., 1998. Multiparameter inversion in anisotropic elastic media, Geophysical Journal International, 134, 757-777.

Candes, E. \& Demanet, L., 2005. The curvelet representation of wave propagators is optimally sparse, Communications on Pure and Applied Mathematics, 58, 1472-1528.

Cao, D., Singh, S., \& Tarantola, A., 1990. Simultaneous inversion for background velocity and impedance maps, Geophysics, $\mathbf{5 5}, 458-469$.

Carazzone, J. \& Srnka, L., 1989. Elastic inversion of Gulf of Mexico data, in Expanded Abstracts, pp. 956-958, Society of Exploration Geophysicists.

Celis, V. \& Larner, K., 2002. Selective-correlation velocity analysis, in Expanded Abstracts, pp. 2289-2292, Society of Exploration Geophysicists.

Chauris, H., 2000. Analyse de vitesse par migration pur l'imagerie des structures complexes en sismique réflexion, $\mathrm{Ph} . \mathrm{D}$. thesis, École des Mines de Paris.

Chauris, H., 2006. Seismic imaging in the curvelet domain and its implications for the curvelet design, in Expanded Abstracts, p. SPMI3.2, Society of Exploration Geophysicists.

Chauris, H. \& Noble, M., 2001. Two-dimensional velocity macro model estimation from seismic reflection data by local differential semblance optimization: applications synthetic and real data sets, Geophysical Journal International, 144, 14-26.

Chavent, G., 1991. New size times curvature conditions for strict quasiconvexity of sets, SIAM Journal on Control and Optimization, 29, 1348-1372.

Chavent, G. \& Lemmonier, P., 1974. Identification de la nonlinéarité d'une équation parabolique quasilinéaire, Applied Mathematics and Optimization, 1, 121-162. 
Claerbout, J.F., 1985. Imaging the Earth's Interior, Blackwell Scientific Publishers, Oxford.

Claerbout, J. \& Doherty, S., 1972. Downward continuation of moveout corrected seismograms, Geophysics, 37, 741-768.

Claerbout, J. F, 1971. Toward a unified theory of reflector mapping, Geophysics, 36, 467-481.

Clément, François \& Chavent, Guy, 1993. Waveform inversion through MBTT formulation, in Mathematical and Numerical Aspects of Wave Propagation, edited by E. Kleinman, T. Angell, D. Colton, F. Santosa, \& I. Stakgold, Society for Industrial and Applied Mathematics, Philadelphia.

Crase, E., Pica, A., Noble, M., McDonald, J., \& Tarantola, A., 1990. Robust elastic nonlinear waveform inversion: Application to real data, Geophysics, 55, 527-538.

de Hoop, M. \& Bleistein, N., 1997. Generalized Radon transform inversions for reflectivity in anisotropic elastic media, Inverse Problems, 16, 669-690.

de Hoop, M. \& Stolk, C., 2002. Microlocal analysis of seismic inverse scattering in anisotropic, elastic media, Communications on Pure and Applied Mathematics, 55, 261-301.

de Hoop, M. \& Stolk, C., 2005. Modeling of seismic data in the downward continuation approach, SIAM Journal on Applied Mathematics, 65, 1388-1406.

de Hoop, M. \& Stolk, C., 2006. Seismic inverse scattering in the downward continuation approach, Wave Motion, 43, 579-598. de Hoop, M., Brandsberg-Dahl, S., \& Ursin, B., 2003. Seismic velocity analysis in the scattering-angle/azimuth domain, Geophysical Prospecting, 51, 295-314.

de Hoop, M., Foss, S.-K., \& Ursin, B., 2005. Depth-consistent reflection tomography using PP and PS seismic data, Geophysics, 70, U51-U65.

Duquet, B. \& Lailly, P., 2006. Efficient 3-d wave-equation migration using virtual planar sources, Geophysics, 71, S185-S197.

Dussaud, E. \& Symes, W., 2005. Velocity analysis from interferometric data, in Expanded Abstracts, p. SPVA1.1, Society of Exploration Geophysicists.

Fernandez-Berdaguer, E. M., Santos, J. E., \& Sheen, D., 1996. An iterative procedure for estimation of variable coefficients in a hyperbolic system, Applied Mathematics and Computation, 76, 213-250.

Gardner, G.H.F., ed., 1985. Migration of Seismic data, Geophysics Reprint Series No. 4, Society of Exploration Geophysicists, , Tulsa.

Gauthier, O., Tarantola, A., \& Virieux, J., 1986. Twodimensional nonlinear inversion of seismic waveforms, Geophysics, 51, 1387-1403.

Gockenbach, M. \& Symes, W., 1999. Coherent noise suppression in velocity inversion, in Expanded Abstracts, pp. 1719-1723, Society of Exploration Geophysicists.

Gockenbach, M.S., Symes, W.W., \& Tapia, R.A., 1995. The dual regularization approach to seismic velocity inversion, Inverse Problems, 11(3), 501-531.

Goupillaud, P., 1961. An approach to inverse filtering of nearsurface layers from seismic records, Geophysics, 26, 754-760.

Gray, S. \& Symes, W.W., 1985. Stability considerations for onedimensional inverse problems, Geophys. J. Roy. Astr. Soc., 80, 149-163.

Jackson, D. D., 1972. Interpretation of inaccurate, insufficient, and inconsistent data, Geophysical Journal of the Royal Astronomical Society, 28, 97-110.

Jackson, D. D., 1976. Most squares inversion, Journal of Geophysical Research, 81, 1027-1030.

Jackson, D. D., 1979. The use of a priori data to resolve nonuniqueness in linear inversion, Geophysical Journal of the Royal Astronomical Society, 57, 137-157.

Jin, S. \& Madariga, 1994. Nonlinear velocity inversion by a twostep Monte-Carlo method, Geophysics, 59, 577-590.

Kern, M. \& Symes, W.W., 1994. Inversion of reflection seismo- grams by differential semblance analysis: Algorithm structure and synthetic examples, Geophysical Prospecting, 99, 565-614.

Khoury, A., Symes, W. W., Williamson, P., \& Shen, P., 2006. DSR migration velocity analysis by differential semblance optimization, in Expanded Abstracts, p. SPMI3.4, Society of Exploration Geophysicists.

Kolb, P., Collino, F., \& Lailly, P., 1986. Prestack inversion of a 1D medium, Proceedings of the IEEE, 74, 498-506.

Lafond, C. F. \& Levander, A. R., 1993. Migration moveout analysis and depth focusing, Geophysics, 58, 91-100.

Lailly, P., 1983. The seismic inverse problem as a sequence of before-stack migrations, in Conference on Inverse Scattering: Theory and Applications, edited by J. Bednar, pp. 206-220, Society for Industrial and Applied Mathematics, Philadelphia. Lailly, P., 1984. Migration methods: partial but efficient solutions to the seismic inverse problem, in Inverse Problems of Acoustic and Elastic Waves, edited by F. Santosa, Society for Industrial and Applied Mathematics, Philadelphia.

Lewis, M.R. \& Symes, W.W., 1991. On the relation between the velocity coefficient and boundary values for solutions of the one-dimensional wave equation, Inverse Problems, 7, 597-632.

Li, J. \& Symes, W., 2005. Fast interval velocity estimation via nmo-based differential semblance, in Expanded Abstracts, p. SPVA1.8, Society of Exploration Geophysicists.

Li, J. \& Symes, W., 2007. Interval velocity estimation via nmobased differential semblance, Geophysics, 72, U75-U88.

Lines, L. \& Treitel, S., 1984. A review of least-squares inversion and its application to geophysical problems, Geophysical Prospecting, 32, 159-186.

Liu, Z. \& Bleistein, N., 1995. Migration velocity analysis: theory and an interative algorithm, Geophysics, 60, 142-153.

Minkoff, S. E. \& Symes, W. W., 1997. Full waveform inversion of marine reflection data in the plane-wave domain, Geophysics, 62(2), 540-553.

Mora, P., 1988. Elastic wavefield inversion of reflection and transmission data, Geophysics, 53, 750-759.

Mosegard, K. \& Tarantola, A., 1991. Monte Carlo analysis of geophysical inverse problems, in Expanded Abstracts, pp. 640643, Society of Exploration Geophysicists.

Mulder, W. \& ten Kroode, A., 2002. Automatic velocity analysis by differential semblance optimization, Geophysics, 67, 11841191.

Neidell, N. \& Taner, T., 1971. Semblance and other coherency measures for multichannel data, Geophysics, 36, 498-509.

Nocedal, J. \& Wright, S., 1999. Numerical Optimization, Springer Verlag, New York.

Nolan, C. \& Symes, W.W., 1997. Global solution of a linearized inverse problem for the wave equation, Communications on Partial Differential Equations, 22, 919-952.

Pao, Y.-H., Santosa, F., \& Symes, W.W., 1984. Inverse problems of acoustic and elastic waves, in Inverse Problems of Acoustic and Elastic Waves, edited by F. Santosa, Society for Industrial and Applied Mathematics, Philadelphia.

Parker, R., 1977. Understanding inverse theory, Annual Review of Earth and Planetary Science, 5, 35-64.

Plessix, R.-E., 2000. Automatic cross-well tomography: an application of the differential semblance optimization to two real examples, Geophysical Prospecting, 48, 937-951.

Plessix, R.-E., 2006. A review of the adjoint-state method for computing the gradient of a functional with geophysical applications, Geophysical Journal International, 167, 495-503.

Plessix, R. E., de Roeck, Y. H., \& Chavent, G., 1995. Automatic and simultaneous migration velocity analysis and waveform inversion of real data using a MBTT/WKBJ formulation, in Expanded Abstracts, pp. 1099-1102, Society of Exploration Geophysicists.

Plessix, R.-E., de Roeck, Y.-H., \& Chavent, G., 1999. Waveform inversion of reflection seismic data for kinematic parameters by 
local optimization, SIAM Journal on Scientific Computation, 20, 1033-1052.

Pratt, R.G, 1999. Seismic waveform inversion in the frequency domain, part 1: Theory, and verification in a physical scale model, Geophysics, 64, 888-901.

Pratt, R.G. \& Shipp, R.M, 1999. Seismic waveform inversion in the frequency domain, part 2: Fault delineation in sediments using crosshole data, Geophysics, 64, 902-914.

Prucha, M., Biondi, B., \& Symes, W., 1999. Angle-domain common image gathers by wave-equation migration, in Expanded Abstracts, pp. 824-827, Society of Exploration Geophysicists.

Rakesh, 1988. A linearized inverse problem for the wave equation, Communications on Partial Differential Equations, 13(5), 573-601.

Rickett, J. \& Sava, P., 2002. Offset and angle-domain common image-point gathers for shot profile migration, Geophysics, 67, 883-889.

Robinson, E.A. \& Treitel, S., 1980. Geophysical Signal Analysis, Prentice-Hall, Englewood Cliffs.

Sacks, P., 1988. The inverse problem for a weakly inhomogeneous two-dimensional medium, SIAM Journal on Applied Mathematics, 48, 1167-1193.

Sacks, P. \& Santosa, F., 1987. A simple computational scheme for determining the sound speed of an acoustic medium from the surface values of its impulse response, SIAM Journal on Scientific and Statistical Computing, 3, 501-520.

Santosa, F. \& Schwetlick, H., 1982. The inversion of acoustic impedance profile by methods of characteristics, Wave Motion, 4, 99-110.

Santosa, F. \& Symes, W.W., 1989. An Analysis of Least-Squares Velocity Inversion, vol. 4 of Geophysical Monographs, Society of Exploration Geophysicists, Tulsa.

Sava, P. \& Fomel, S., 2003. Angle-domain common-image gathers by wavefield continuation methods, Geophysics, 68, 10651074 .

Sava, P. \& Fomel, S., 2005. Time-shift imaging condition, in Expanded Abstracts, pp. SPMI2-2, Society of Exploration Geophysicists.

Sen, M.K. \& Stoffa, P., 1991. Nonlinear one-dimensional seismic waveform inversion using simulated annealing, Geophysics, $\mathbf{5 6}$, 1624-1636.

Sen, M.K. \& Stoffa, P., 1991. Nonlinear multiparameter optimization using genetic algorithms: Inversion of plane wave seismograms, Geophysics, 56, 1794-1810.

Shen, P. \& Calandra, H., 2005. One-way waveform inversion within the framework of adjoint state differential migration, in Expanded Abstracts, p. SI3.5, Society of Exploration Geophysicists.

Shen, P., Symes, W., \& Stolk, C., 2003. Differential semblance velocity analysis by wave-equation migration, in Expanded $\mathrm{Ab}$ stracts, pp. 2135-2139, Society of Exploration Geophysicists.

Shen, P., Menyoli, E., \& Calandra, H., 2005. Automatic subsalt velocity analysis: an integrated strategy of ray-based tomography and wave-equation migration velocity inversion, in Expanded Abstracts, p. SPMI6.3, Society of Exploration Geophysicists.

Shen, P., Symes, W., Morton, S., \& Calandra, H., 2005. Differential semblance velocity analysis via shot profile migration, in Expanded Abstracts, p. SPVA1.4, Society of Exploration Geophysicists.

Shin, C. \& Min, D.-J., 2006. Waveform inversion using a logarithmic wavefield, Geophysics, 71, R31-R42.

Sirgue, L. \& Pratt, G., 2004. Efficient waveform inversion and imaging: a strategy for selecting temporal frequencies, Geophysics, 69, 231-248.

Soubaras, R. \& Gratacos, B., 2006. Velocity model building by semblance maximization of modulated-shot gathers, in Expanded Abstracts, p. SVIP1.3, Society of Exploration Geo- physicists.

Stolk, C., 2000. On the modeling and inversion of seismic data, Ph.D. thesis, Universiteit Utrecht.

Stolk, C., 2000. Microlocal analysis of a seismic linearized inverse problem, Wave Motion, 32, 267-290.

Stolk, C. C. \& Symes, W. W., 2003. Smooth objective functionals for seismic velocity inversion, Inverse Problems, 19, 73-89.

Stolk, C. C. \& Symes, W. W., 2004. Kinematic artifacts in prestack depth migration, Geophysics, 69, 562-575.

Symes, W.W., 1980. Numerical stability in an inverse scattering problem, SIAM Journal on Numerical Analysis, 17(5), MRC TSR 1990, (August 1979).

Symes, W.W., 1983. Impedance profile inversion via the first transport equation, J. Math. Anal. Appl., 94, 435-453.

Symes, W.W., 1986. On the relation between coefficient and boundary values for solutions of webster's horn equation, SIAM Journal on Mathematical Analysis, 17, 1400-1420.

Symes, W.W., 1986. Stability and instability results for inverse problems in several-dimensional wave propagation, in Proc. 7th International Conference on Computing Methods in Applied Science and Engineering, edited by R. Glowinski \& J. Lions, North-Holland.

Symes, W.W., 1990. Velocity inversion: A case study in infinitedimensional optimization, Mathematical Programming, 48, $71-102$.

Symes, W.W., 1991. Layered velocity inversion: a model problem from reflection seismology, SIAM Journal on Mathematical Analysis, 22, 680-716.

Symes, W.W., 1991. A differential semblance algorithm for the inverse problem of reflection seismology, Computers and Mathematics with Applications, 22(4/5), 147-178.

Symes, W.W., 1991. Non-interactive estimation of the Marmousi velocity model by differential semblance optimization: Initial trials, in The Marmousi Experience: Proceedings of the EAEG Workshop on Practical Aspects of Inversion, edited by G. Grau \& R. Versteeg, IFP/Technip.

Symes, W.W., 1993. A differential semblance criterion for inversion of multioffset seismic reflection data, Journal of Geophysical Research, 98, 2061-2073.

Symes, W., 1995. Mathematical Foundations of Reflection Seismology, Tech. rep., Department of Computational and Applied Mathematics, Rice University, Houston, Texas, USA, www.trip.caam.rice.edu.

Symes, W.W. \& Carazzone, J., 1991. Velocity inversion by differential semblance optimization, Geophysics, 56(5), 654-663.

Symes, W.W. \& Carazzone, J.J., 1992. Velocity inversion by coherency optimization, in Geophysical Inversion, edited by J. Bednar, pp. 59-89, Society for Industrial and Applied Mathematics, Philadelphia.

Symes, W.W. \& Gockenbach, M., 1995. Waveform inversion for velocity: Where have all the minima gone?, in Expanded $A b$ stracts, pp. 1235-1239.

Symes, W.W. \& Versteeg, R., 1993. Velocity model determination using differential semblance optimization, in Expanded Abstracts, pp. 696-699, Society of Exploration Geophysicists, Tulsa, OK.

Symes, W. W., 1998. High frequency asymptotics, differential semblance, and velocity analysis, in Expanded Abstracts, pp. 1616-1619, Society of Exploration Geophysicists.

Symes, W. W., 1999. All stationary points of differential semblance are asymptotic global minimizers: layered acoustics, Tech. Rep. 99-29, Department of Computational and Applied Mathematics, Rice University, Houston, Texas, USA.

Symes, W. W., 2001. Asymptotic analysis of differential semblance for layered acoustics, Tech. Rep. 01-21, Department of Computational and Applied Mathematics, Rice University, Houston, Texas, USA.

Symes, W. W., 2002. Kinematics of reverse time shot-geophone 
migration, The Rice Inversion Project, Department of Computational and Applied Mathematics, Rice University, Houston, Texas, USA: http://www.trip.caam.rice.edu.

Taner, M.T. \& Koehler, F., 1969. Velocity spectra: digital computer derivation and application of velocity functions, Geophysics, 34, 859-881.

Tarantola, A., 1984. Inversion of seismic reflection data in the acoustic approximation, Geophysics, 49, 1259-1266.

Tarantola, A., 1987. Inverse Problem Theory, Elsevier.

Tarantola, A., Crase, E., Jervis, M., Konen, Z., Lindgren, J., Mosegard, K., \& Noble, M., 1990. Nonlinear inversion of seismograms: State of the art, in Expanded Abstracts, p. S13.7, Society of Exploration Geophysicists.

Taylor, M., 1981. Pseudodifferential Operators, Princeton University Press, Princeton, New Jersey.

Ten Kroode, A. P. E., Smit, D. J., \& Verdel, A. R., 1998. A microlocal analysis of migration, Wave Motion, 28, 149-172.

Toldi, J., 1989. Velocity analysis without picking, Geophysics, 54, 191-199.

Treitel, S., Gutowski, P.R., \& Wagner, D.E., 1982. Plane-wave decomposition of seismograms, Geophysics, 47, 1375-1401.

Verm, R. \& Symes, W., 2006. Practice and pitfalls in NMObased differential semblance velocity analysis, in Expanded $A b$ stracts, p. SI3.7, Society of Exploration Geophysicists.

$\mathrm{Xu}$, Sheng, Chauris, Hervé, Lambaré, Gilles, \& Noble, Mark, 2001. Common angle migration: A strategy for imaging complex media, Geophysics, 66(6), 1877-1894.

Yilmaz, O., 2001. Seismic data processing, in Investigations in Geophysics No. 10, Society of Exploration Geophysicists, Tulsa.

\section{FIGURE CAPTIONS}

Figure 1. A simple three layer velocity model.

Figure 2. Common shot gather for velocity model of Figure 1. Source wavelet is $(4,10,30,40) \mathrm{Hz}$ trapezoidal zero-phase bandpass filter.

Figure 3. Results of three trials of output least squares inversion for the data of Figure 2. In each case (red, green, blue), the dashed line is the initial velocity estimate, the wiggly line is the final estimate. Limited memory BroydenFletcher-Goldfarb-Shanno iteration halted when the gradient norm dropped to $1 \%$ of its initial value. Note that (a) only when the initial velocity is correct up to the second interface (blue lines) is the estimate of the second interface position correct; (b) the surface multiple from the second interface shows up as an inhomogeneity in the estimated velocity for both the constant velocity initial estimate (red lines) and the estimate with a second layer at $2.0 \mathrm{~km} / \mathrm{s}$ (green lines), but disappears when the kinematics are correct (blue lines); (c) the iteration fails in both of the first two cases to update the second layer mean velocity; (d) in all cases the velocity below the second interface remains at its initial value, because no constraint is present in the data.

Figure 4. Inversion for extended model, constant initial velocity ( $\equiv 1.5 \mathrm{~km} / \mathrm{s}$, red dashed line in Figure 3 ). Note the large amount of energy spread across the entire offset range. Figure 5. Inversion for extended model, initial velocity includes jump to $2 \mathrm{~km} / \mathrm{s}$ at depth of $0.2 \mathrm{~km}$ (green dashed line in Figure 3). Energy still present at nonzero offsets, but less so than in Figure 4.

Figure 6. Inversion for extended model, initial velocity includes jump to $2.4 \mathrm{~km} / \mathrm{s}$ at depth of $0.2 \mathrm{~km}$ (blue dashed line in Figure 3), which is kinematically correct down to the second interface. Energy mostly focused at zero offset, except for inversion noise which should be suppressed by proper regularization. Note that second reflector now appears at correct depth, and multiple reflection (visible in Figures 4 and 5) has disappeared.

Figure 7. Resimulated data from inverted model of Figure 4.

Figure 8. Resimulated data from inverted model of Figure 5.

Figure 9. Resimulated data from inverted model of Figure 6. 

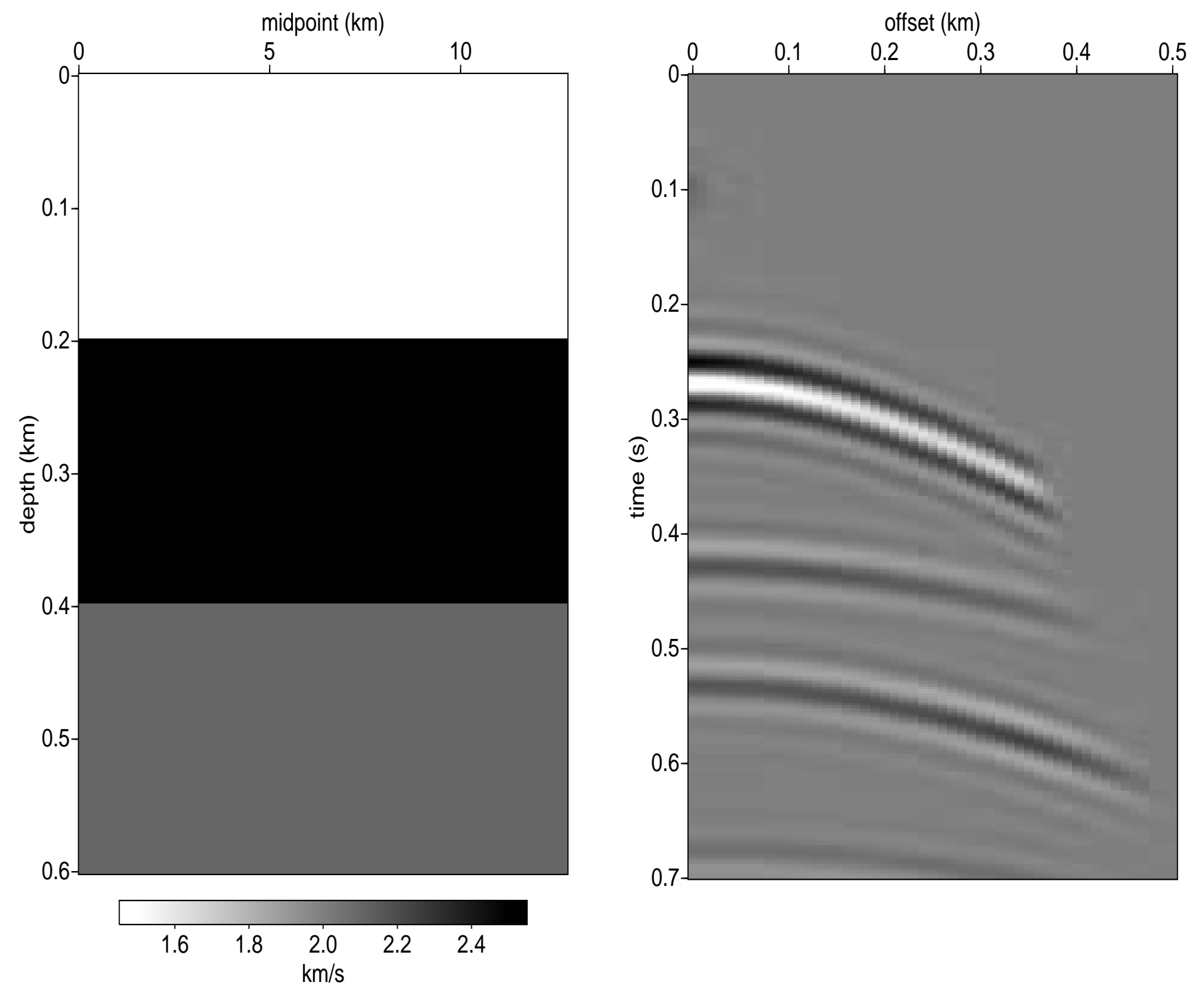

Figure 1.

Figure 2. 

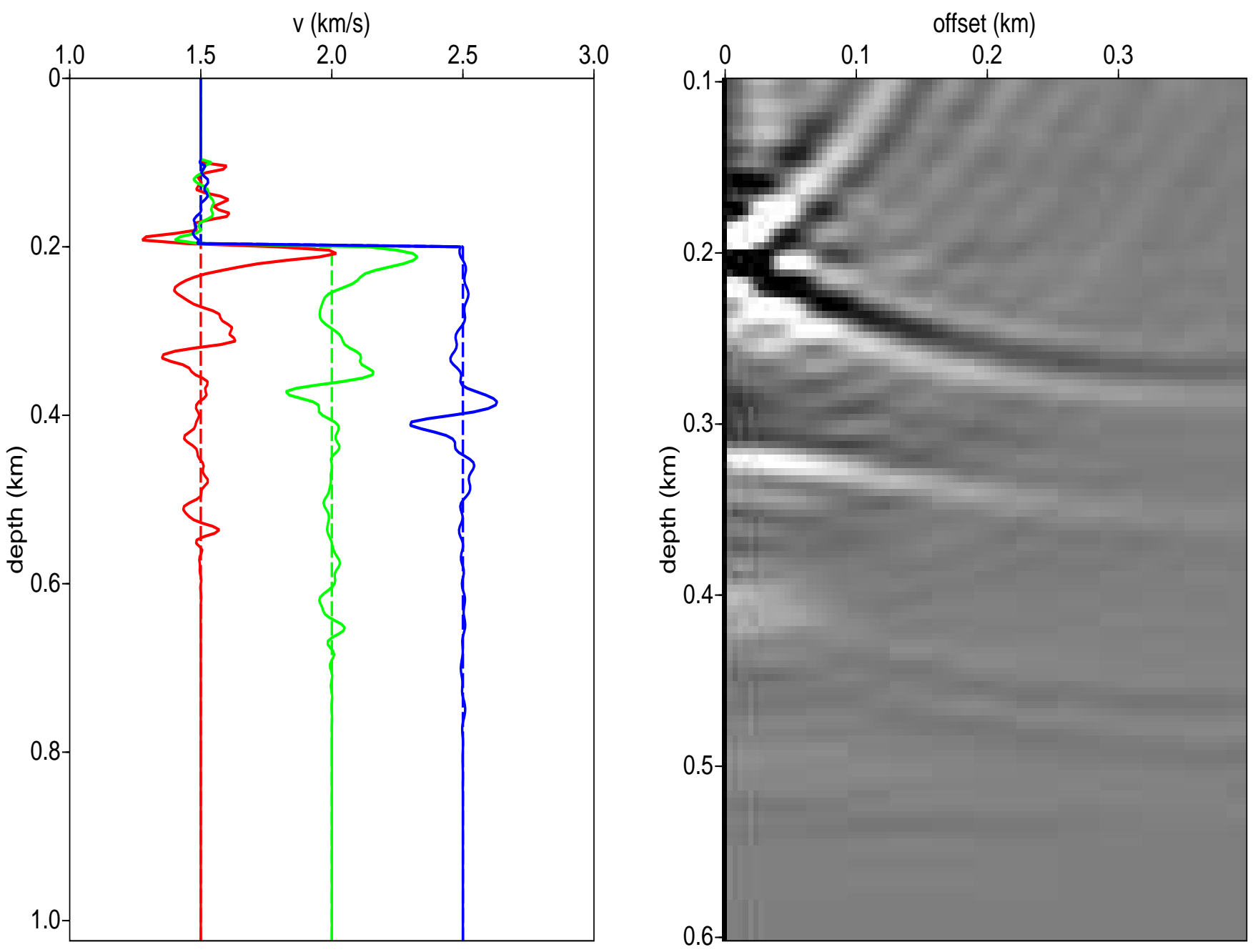
22 W. Symes
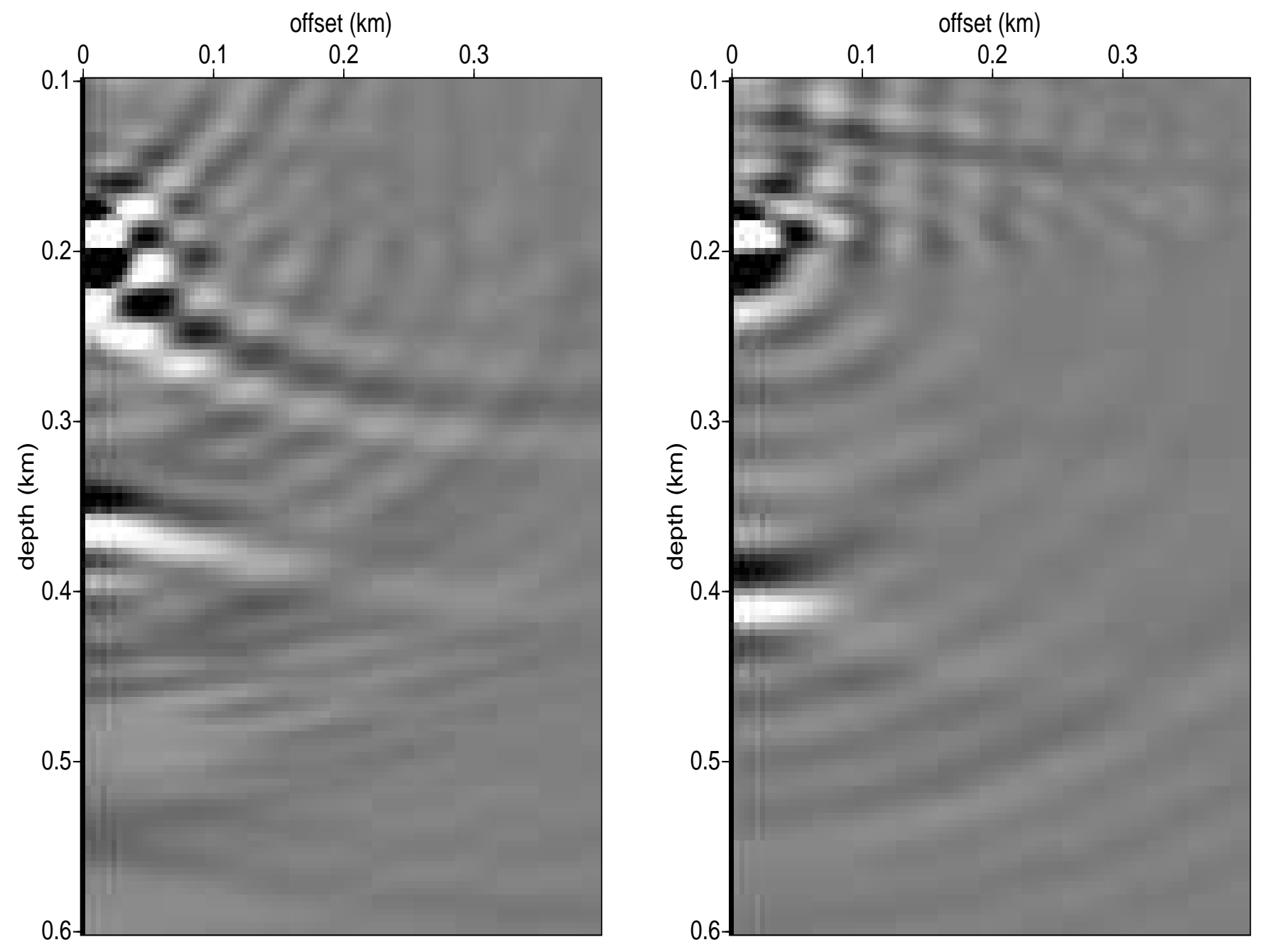

Figure 5.

Figure 6. 

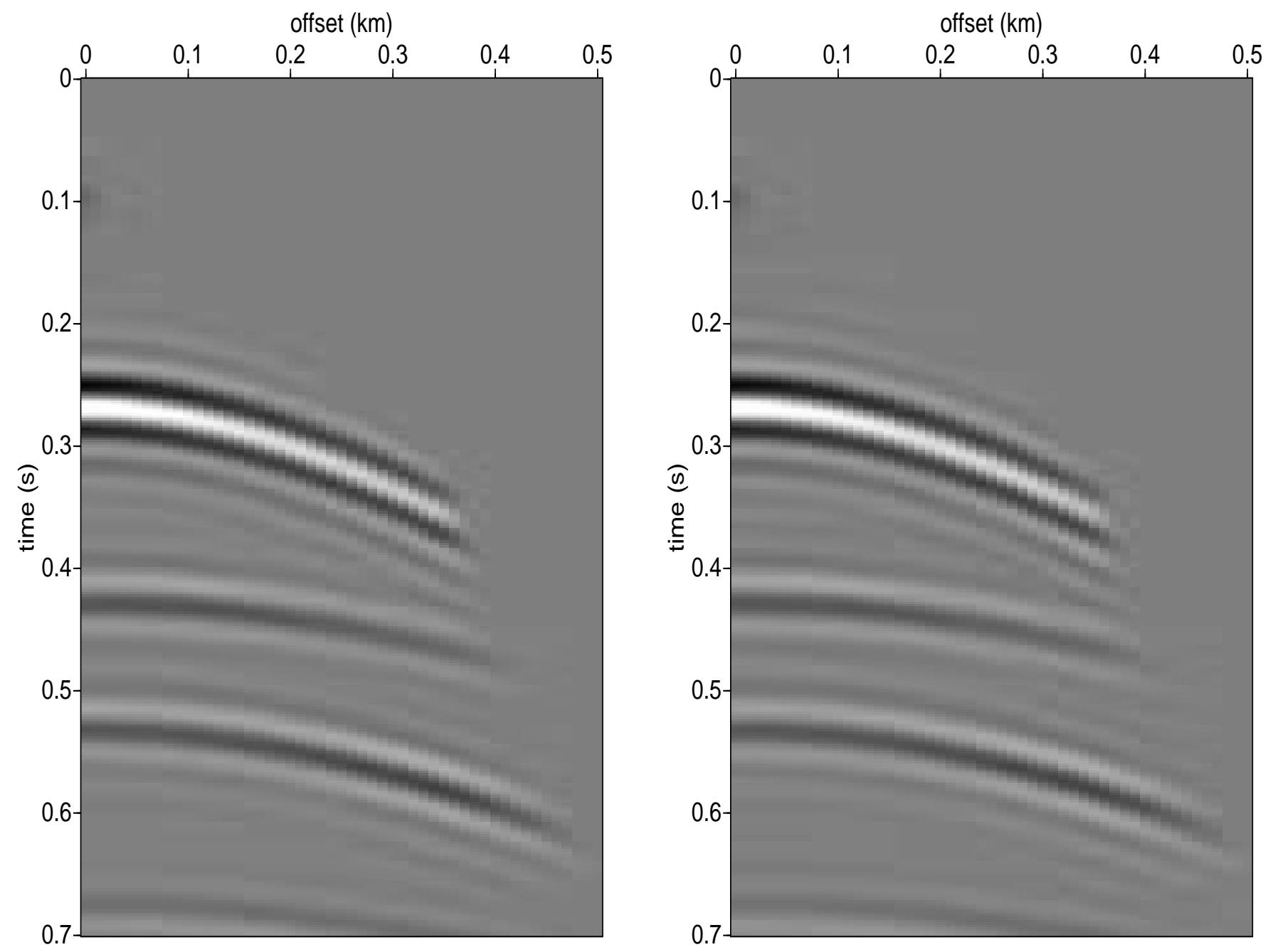
24 W. Symes

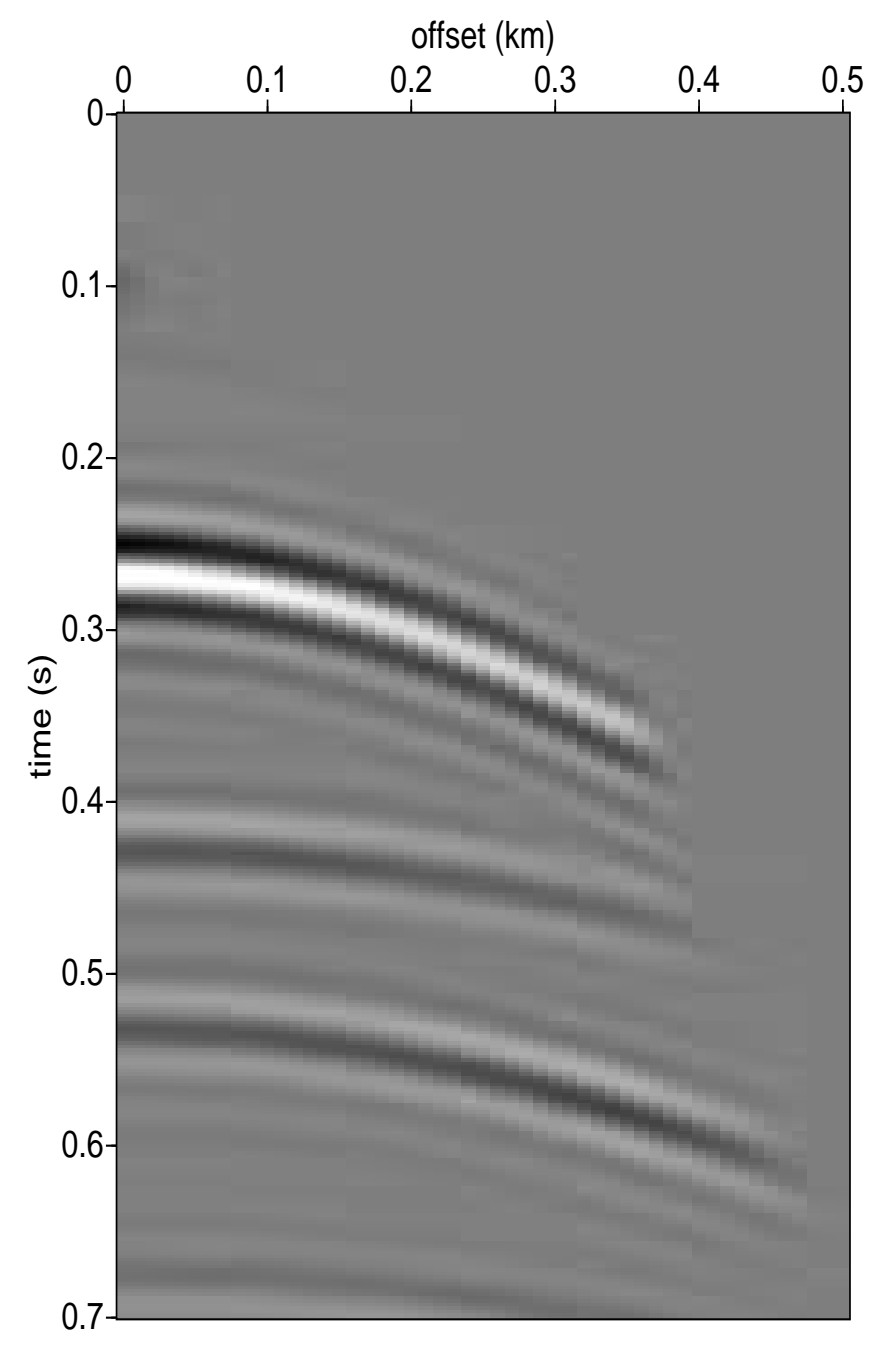

Figure 9. 